\title{
Mechanisms of aortic dissection smooth muscle cell phenotype switch
}

\author{
Zhao An, MD, Yang Liu, MD, Zhi-Gang Song, MD, Hao Tang, MD, Yang Yuan, MD, and Zhi-Yun Xu, MD
}

\section{ABSTRACT}

Objective: To investigate the expression of Nanog homeobox (NANOG) in thoracic aortic dissection (TAD) and the role of NANOG in regulating human aortic vascular smooth muscle cells (VSMCs) phenotype switch.

Methods: Aortic specimens were collected from 20 patients undergoing TAD and 10 controls. VSMCs were isolated by adherent cultivation approach. The expression of NANOG, osteopontin (OPN), and VSMCs phenotype markers were determined by quantitative real-time polymerase chain reaction, Western blot, immunohistochemistry, and immunofluorescence. Cell counting, scratch wound-healing assay, Transwell migration, and apoptosis assays were used for cell function assessment. Deoxyribonucleic acid-protein binding detection was performed by chromatin immunoprecipitation.

Results: Our experiment results showed that NANOG and OPN were highly expressed in TAD aortic wall and VSMCs, both accompanying VSMCs phenotype switch. Overexpression of NANOG induced the up-regulation of VSMCs synthetic marker matrix metalloproteinase 2 and the down-regulation of VSMCs contractile markers $\alpha$-smooth muscle actin and smooth muscle $22 \alpha$. Overexpression of NANOG also enhanced the proliferation, migration, and antiapoptosis capabilities of VSMCs. The results also showed that these functions of NANOG was via OPN and NANOG directly up-regulated OPN by binding to its promoter region.

Conclusions: Our study suggests that NANOG is highly expressed in TAD aortic wall and VSMCs. Increased NANOG promotes VSMCs phenotype switch by directly up-regulating OPN through binding to its promoter region. (J Thorac Cardiovasc Surg 2017;154:1511-21)

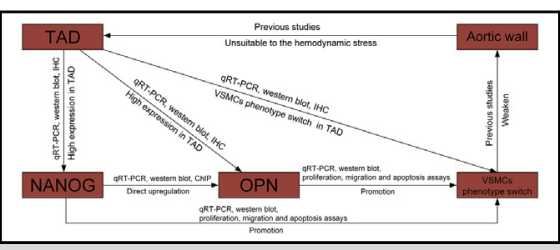

Expression of Nanog homeobox (NANOG) and OPN was increased in TAD accompanying vascular smooth muscle cells (VSMCs) phenotype switch. Overexpression of NANOG promoted VSMCs phenotype switch by directly up-regulating OPN

\section{Central Message}

Nanog homeobox is highly expressed in thoracic aortic dissection and promotes the vascular smooth muscle cells phenotype switch by directly up-regulating osteopontin through binding to its promoter region.

\section{Perspective}

Thoracic aortic dissection (TAD) is a lifethreating disease. Although the treatment strategy of it has been improved continuously, the mortality of TAD is still very high and its pathogenesis has not been clarified fully. This study aimed to clarify the mechanism responsible for the occurrence of TAD.

See Editorial Commentary page 1522.
Thoracic aortic dissection (TAD) is a life-threating medical emergency with very high mortality. ${ }^{1,2}$ Some connective tissue diseases can result in TAD, but only approximately $10 \%$ of TAD can be attributed to connective tissue diseases, and the pathogenesis of the others is still unclear. ${ }^{3}$ It is known that the abnormity of aortic media is

\footnotetext{
From the Department of Cardiovascular Surgery, Changhai Hospital, Second Military Medical University, Shanghai, China.

Z.A. and Y.L. contributed equally to this work.

This work was supported by Shanghai Committee of Science and Technology (grant 16ZR1400900 to Dr Liu)

Received for publication July 22, 2016; revisions received May 1, 2017; accepted for publication May 18, 2017; available ahead of print June 16, 2017.

Address for reprints: Yang Yuan, MD, or Zhi-Yun Xu, MD, Department of Cardiovascular Surgery, Changhai Hospital, Second Military Medical University, 168 Changhai Rd, Shanghai 200433, China (E-mail: yangyuan21@yeah.net or zhiyunx@hotmail.com).

$0022-5223 / \$ 36.00$

Copyright (c) 2017 by The American Association for Thoracic Surgery

http://dx.doi.org/10.1016/j.jtcvs.2017.05.066
}

the pathogenetic base of TAD. ${ }^{4,5}$ Vascular smooth muscle cells (VSMCs) are the main cell type of aortic media and can transform between contractile and synthetic phenotypes. ${ }^{6}$ Compared with contractile VSMCs, synthetic VSMCs show the enhanced proliferation, migration, and expression of extracellular matrix components like matrix metalloproteinase 2 (MMP2) and matrix metalloproteinase 9, but synthetic VSMCs express decreased levels of contractile markers such as $\alpha$-smooth muscle actin $\left(\alpha\right.$-SMA) and smooth muscle $22 \alpha(\operatorname{SM} 22 \alpha){ }^{7}$ It was reported the VSMCs phenotype switch might be involved

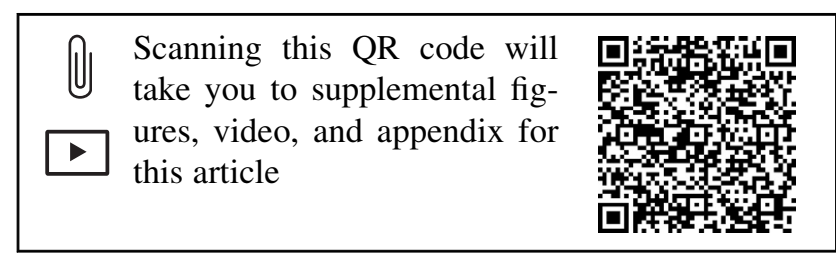




\begin{tabular}{|c|c|}
\hline \multicolumn{2}{|c|}{ Abbreviations and Acronyms } \\
\hline & $\mathrm{i}=$ NANOG overexpressiol \\
\hline$\alpha$-SMA & $=\alpha$-smooth muscle actin \\
\hline ChIP & $=$ chromatin immunoprecipitation \\
\hline MMP2 & $=$ matrix metalloproteinase 2 \\
\hline NANOG & $=$ Nanog homeobox \\
\hline OPN & $=$ osteopontin \\
\hline qRT-PCR & $\begin{array}{l}=\text { quantitative real-time polymerase } \\
\text { chain reaction }\end{array}$ \\
\hline si-NANOG & $\begin{array}{l}=\text { NANOG-specific small interfering } \\
\text { RNA }\end{array}$ \\
\hline si-NC & $=$ scrambled negative control siRNA \\
\hline si-OPN & $\begin{aligned}= & \text { OPN-specific small interfering RNA } \\
& \text { adenovirus }\end{aligned}$ \\
\hline $\operatorname{SM} 22 \alpha$ & $=$ smooth muscle $22 \alpha$ \\
\hline TAD & $=$ thoracic aortic dissection \\
\hline TUNEL & $\begin{aligned}= & \text { terminal deoxynucleotidyl } \\
& \text { transferase dUTP nick end labeling }\end{aligned}$ \\
\hline UV & $=$ ultraviolet \\
\hline VSMC & $=$ vascular smooth muscle cell \\
\hline
\end{tabular}

in the pathophysiologic processes of TAD.$^{8-11}$ However, little is known about the molecular mechanism of the VSMCs phenotype switch in TAD.

Osteopontin (OPN), also known as secreted phosphoprotein 1 , is a multifunction protein that is highly expressed in synthetic VSMCs. ${ }^{9}$ OPN is related to some important biological behaviors of VSMCs, and high-level OPN promotes the proliferation and migration of VSMCs. ${ }^{12}$ In addition, OPN also contributes to the apoptotic resistance of VSMCs. ${ }^{13}$ It was reported OPN up-regulates the expression of MMP2 and down-regulates the expression of $\alpha$-SMA in VSMCs. ${ }^{14-16}$ Moreover, Wang and colleagues $^{8}$ reported OPN was highly expressed in TAD aortic media accompanying the VSMCs phenotype switch. All these findings indicate that OPN may induce the VSMCs phenotype switch in the pathophysiologic processes of TAD. However, there are no reports on the exact mechanism of OPN up-regulation in TAD.

Nanog homeobox (NANOG) is a homeodomain protein and has a vital function in the transcriptional network of pluripotency and proliferation in embryonic stem cells. The expression of NANOG maintains the pluripotency and self-renew of embryonic stem cells and induces cell dedifferentiation. ${ }^{17-19}$ In contrast, deficiency of NANOG induces cell differentiation. ${ }^{20}$ The function of NANOG suggests it may be related with the phenotype switch of VSMCs in TAD, which is regarded as a dedifferentiation process. ${ }^{11,21}$ More importantly, it was reported NANOG might regulate the expression of OPN in human embryonic stem cells. ${ }^{22}$
All these indicate that the up-regulation of OPN in TAD may be regulated by NANOG.

In this study, by a series of histology and molecular biology experiments, we sought to determine whether NANOG promoted the VSMCs phenotype switch by directly up-regulating OPN in the pathophysiologic processes of TAD.

\section{METHODS}

\section{Specimen Collection}

Specimens used in this study were acquired at the Department of Cardiovascular Surgery, Changhai Hospital, from April to June, 2015. Dissected aortic specimens near the crevasse were obtained from the rupture area in 20 continuous patients who consented to undergo TAD repair surgery. Patients with traumatic aortic injury, inflammatory aortic disease, Ehlers-Danlos syndrome, Marfan syndrome, and other connective tissue disorders were excluded. Control aortic specimens were obtained from 10 continuous patients who underwent aortic valve replacement and who had no vascular diseases. Table E1 summarizes patient details. Specimens were divided into pieces and preserved under different conditions for cell culture, histologic, and biochemical analysis. This study conformed to the principles outlined in the Declaration of Helsinki, and informed consent was obtained from all patients or their direct relatives. This study was approved by the Institutional Review Board of Changhai Hospital.

\section{Cell Culture}

Primary VSMCs were isolated from aortic media by an adherent cultivation approach (Video 1 and Appendix E1).

\section{Genes Overexpression and Silencing in VSMCs}

NANOG overexpression adenovirus (Ad-NANOG) with green fluorescent protein tag and green fluorescent protein control adenovirus were purchased from Hanbio Biotechnology (Shanghai, China). NANOG-specific small interfering RNA (si-NANOG) and the scrambled

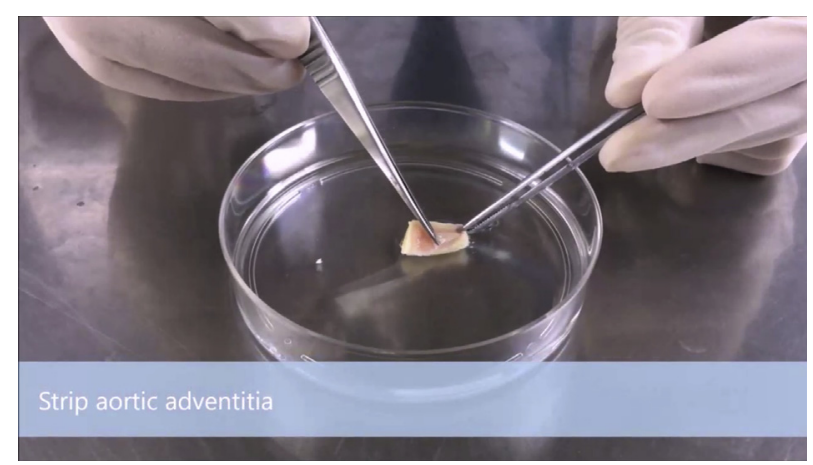

VIDEO 1. Isolation of aortic vascular smooth muscle cells by adherent cultivation approach. First, aortic adventitia and intima were stripped from specimens. Then, the rest were cut into about $4-\mathrm{mm}^{2}$ tissue blocks and each block was inoculated in a 24-well culture plate. The plate was inversed and incubated at $37^{\circ} \mathrm{C}$ with $5.0 \%$ carbon dioxide for 1 hour. After that, $200 \mu \mathrm{L}$ of Medium 231 with smooth muscle growth supplements (Cascade Biologics, Waltham, Mass) was added into each well. At last, the plate was incubated at $37^{\circ} \mathrm{C}$ with $5.0 \%$ carbon dioxide and the culture medium was replaced every 72 hours. Video available at: http://www.jtcvsonline. org/article/S0022-5223(17)31119-4/addons. 


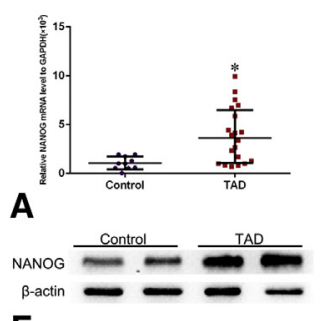

$\mathbf{F}$

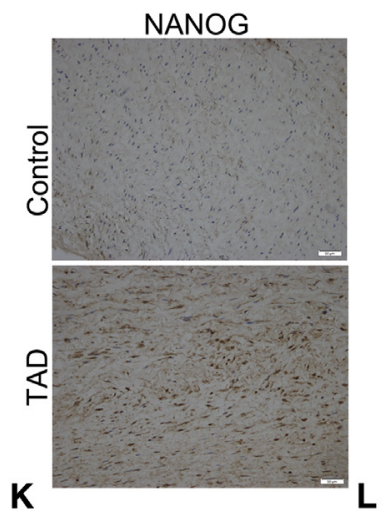

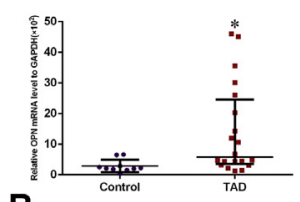

B

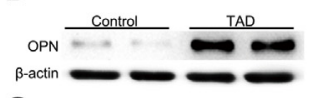

G

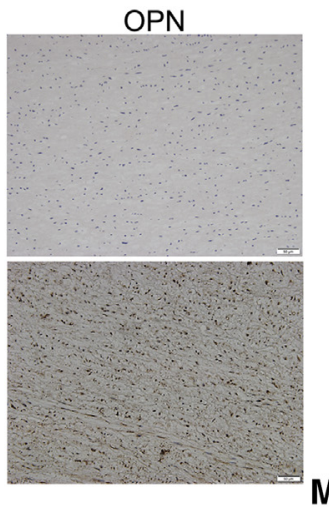

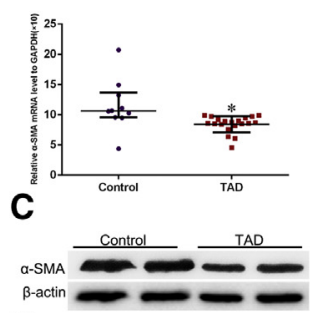

H

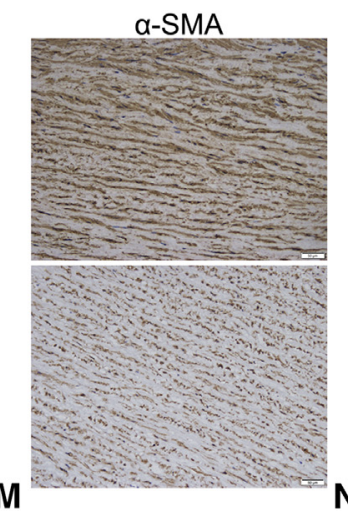

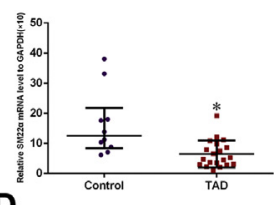

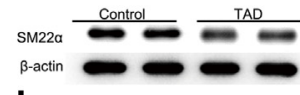

I

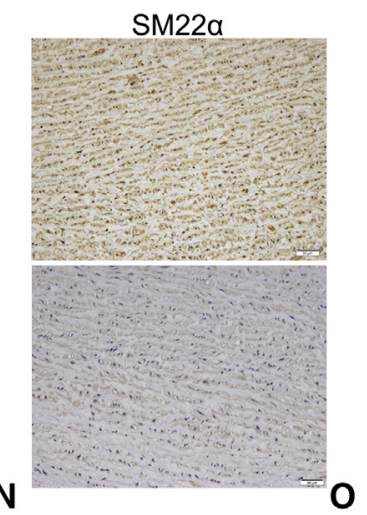

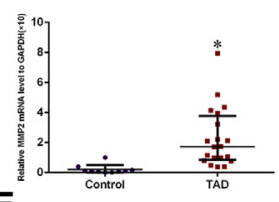

E

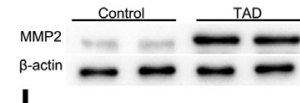

MMP2

FIGURE 1. The expression of NANOG, OPN, and VSMCs phenotype markers in the aortic media of TAD and control patients. A-E, Quantitative real-time polymerase chain reaction results showed that NANOG and OPN mRNA levels were higher (A and B), mRNA levels of VSMC contractile markers $\alpha$-SMA (C) and SM22 $\alpha$ (D) were lower whereas mRNA levels of (E) synthetic MMP2 was greater in the TAD group $(n=20)$ compared with the control group $(n=10)$. F-J, Typical Western blot images showed that protein levels of (F) NANOG and (G) OPN were significantly greater, protein levels of VSMCs contractile markers (H) $\alpha$-SMA and (I) SM22 $\alpha$ were decreased and protein level of VSMCs synthetic marker (J) MMP2 was elevated in aortic media specimens from patients with TAD. $\beta$-actin was used as the internal reference. K-O, Typical immunohistochemical images of NANOG, OPN, and VSMC phenotype markers in TAD and control aortic media specimens $(\times 200)$. Error bars represent the median, $25 \%$ and $75 \%$ quartiles. Student $t$ test and Mann-Whitney $U$ test were used to compare differences between 2 groups. ${ }^{*} P<.05$ versus control. NANOG, Nanog homeobox; GAPDH, glyceraldehyde-3-phosphate dehydrogenase; $T A D$, thoracic aortic dissection; OPN, osteopontin; $\alpha$-SMA, $\alpha$-smooth muscle actin; SM22 $\alpha$, smooth muscle $22 \alpha ; M M P 2$, marker matrix metalloproteinase 2.

negative control small interfering RNA (si-NC) were purchased from GenePharma (Shanghai, China). OPN-specific small interfering RNA adenovirus (si-OPN) and si-NC were purchased from Obio Technology (Shanghai, China). Lipofectamine 2000 (Invitrogen, Life Technologies, Carlsbad, Calif) was used for NANOG interference according to the manufacturer's instructions.

\section{Immunohistochemistry}

Operation procedures was performed as described previously. ${ }^{23}$ Primary antibodies including anti-NANOG (4903S; Cell Signaling Technology, Boston, Mass), anti-OPN (BS1264; Bioworld Technology, Minneapolis, Minn), anti- $\alpha$-SMA (BM0002; Boster Technology, Wuhan, China), anti-SM22 $\alpha$ (ab14106; Abcam, Cambridge, United Kingdom), and anti-MMP2 (ab92536; Abcam, Cambridge, United Kingdom) were used for protein detection. Six specimens from each group were made into paraffin sections. Positive signals were semiquantified with ImageJ software (National Institutes of Health, Bethesda, Md).

\section{Quantitative Real-Time Polymerase Chain Reaction (qRT-PCR)}

qRT-PCR was performed as described previously, ${ }^{11,24,25}$ with modifications (Appendix E1).

\section{Western Blot}

Tissue specimens used for Western blot detection were stripped the intima and adventitia. The procedure of Western blot was the same as described previously. ${ }^{26}$ Primary antibodies used in this part were the same with immunohistochemical staining. The band intensity was quantified by densitometric measurement with ImageJ software (National Institutes of Health) and normalized to the expression level of $\beta$-actin.

\section{Cell Proliferation}

Cell Counting Kit-8 (CCK8, Beyotime, Haimen, Shanghai, China) was used for the assessment of cell proliferation. A 96-well plate was used for the assay, and the detailed procedures were performed as per our previous description, with modifications. ${ }^{24} \mathrm{~A}$ total of $10 \mu \mathrm{L}$ of CCK8 solution was added at $0,24,48,72$, and 96 hours after adenovirus transfection. Then, the cultures were incubated at $37^{\circ} \mathrm{C}$ with $5.0 \%$ carbon dioxide for 1.5 hours. After that, absorbance was measured at $450 \mathrm{~nm}$.

\section{Scratch Wound-Healing Assay and Transwell Migration Assay}

Scratch wound-healing assay and Transwell migration assay were used for cell migration assessment. The experiments were performed as described previously. ${ }^{24}$

\section{Apoptosis Assay}

Ultraviolet (UV) irradiation was adopted to induce VSMCs apoptosis, which was established elsewhere. ${ }^{27}$ In brief, VSMCs were seeded in a 6-well plate $\left(2 \times 10^{5} /\right.$ well). At 48 hours after transfection, the culture medium was replaced with serum-free medium for 4 hours to synchronize cells. The medium was then changed back to M231 with smooth muscle growth supplements for 2 hours. After that, VSMCs were exposed to UV 

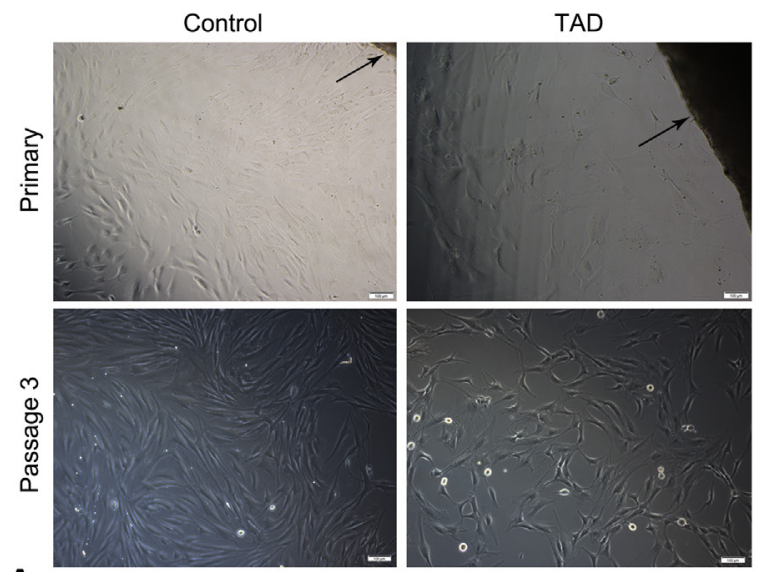

A
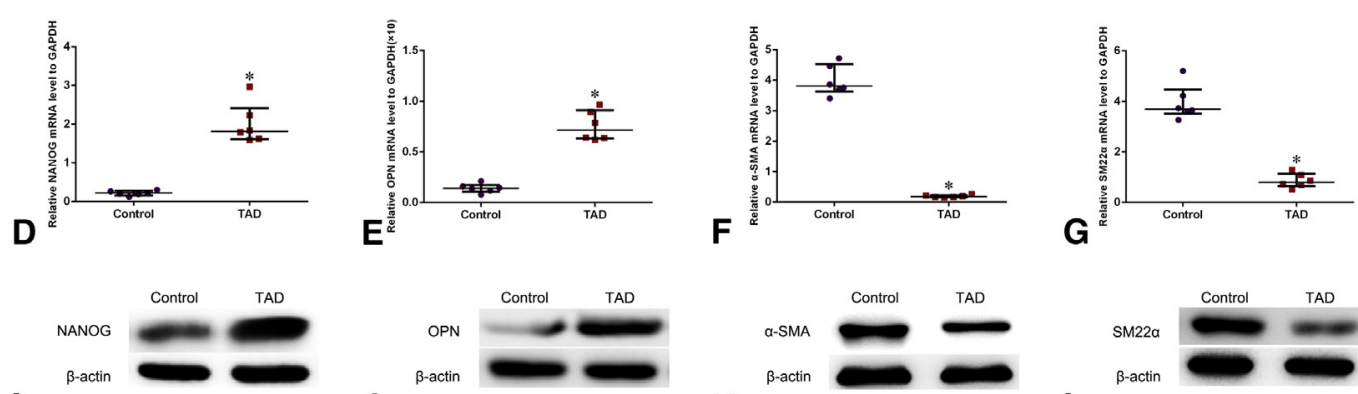

I
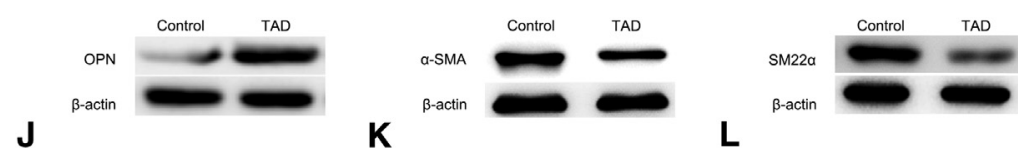

H
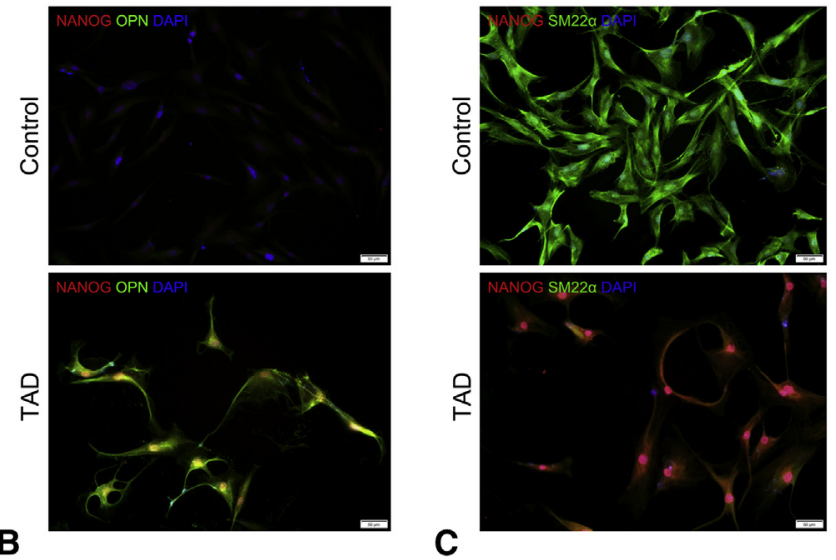

K

$\mathbf{L}$

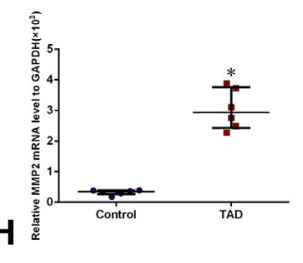

FIGURE 2. VSMCs were isolated from aortic specimens of TAD and control patients and the expression of NANOG, OPN, and VSMCs phenotype markers on mRNA and protein levels were detected. A, Primary VSMCs were isolated by adherent cultivation approach, VSMCs isolated from patients with TAD lost the elongated shape compared with controls $(\times 100)$. Tissue block was pointed by arrows. B, Immunofluorescent analysis of NANOG and OPN in VSMCs isolated from TAD and control specimens, the staining for NANOG (red) and OPN (green) was enhanced in nucleus and cytoplasm of TAD VSMCs, respectively $(\times 200)$. The nucleus was stained as blue with DAPI. C, Immunofluorescent analysis of NANOG and SM $22 \alpha$ in VSMCs isolated from TAD and control specimens, the staining for NANOG (red) was enhanced and SM22 $\alpha$ (green) was decreased in TAD VSMCs $(\times 200)$. The nucleus was stained as blue with DAPI. D-H, The expression of (D) NANOG and (E) OPN on mRNA level was increased, mRNA levels of VSMCs contractile markers (F) $\alpha$-SMA and (G) SM22 $\alpha$ were decreased and the expression of $(\mathrm{H})$ synthetic MMP2 was increased in TAD VSMCs $(n=6)$ compared with controls $(n=6)$. I-M, Typical Western blot images showed that protein level of (I) NANOG and (J) OPN were elevated, contractile markers (K) $\alpha$-SMA and (L) SM22 $\alpha$ were decreased and (M) synthetic marker MMP2 was increased in TAD VSMCs compared with controls. $\beta$-actin was used as the internal reference in western blot. Error bars represent the median, $25 \%$ and $75 \%$ quartiles. The Student $t$ test was used to compare differences between two groups. $* P<.05$ versus control. TAD, Thoracic aortic dissection; NANOG, Nanog homeobox; OPN, osteopontin; DAPI, 4',6-diamidino-2-phenylindole; SM22 $\alpha$, smooth muscle 22 $\alpha$; GAPDH, glyceraldehyde-3-phosphate dehydrogenase; $\alpha$-SMA, $\alpha$-smooth muscle actin; MMP2, marker matrix metalloproteinase 2.

for 15 minutes and then incubated at $37^{\circ} \mathrm{C}$ with $5.0 \%$ carbon dioxide for 6 hours. At last, cells were harvested and detected. Flow cytometry with Annexin V-APC/PI Apoptosis Kit (eBioscience, San Diego, Calif) was used for apoptosis assay according to the manufacturer's instructions. FlowJo software (TreeStar, Ashland, Ore) was used for data analysis.

\section{Terminal Deoxynucleotidyl Transferase dUTP Nick End Labeling (TUNEL) Assay}

A TUNEL assay was performed to detect apoptosis induced by UV (Appendix E1).

\section{Immunofluorescence}

Immunofluorescence assay was performed as described previously, ${ }^{11}$ with modifications (Appendix E1).

\section{Chromatin Immunoprecipitation (ChIP)}

A ChIP assay was performed to detect the NANOG binding site in the promoter region of OPN (Appendix E1).

\section{Statistical Analysis}

Statistical Package for Social Sciences, version 22.0 (SPSS, Chicago, Ill) was used for data analysis. For continuous variables, the Kolmogorov-Smirnov test was used to examine whether the data were distributed normally. Statistical differences were evaluated by Student $t$ test when the data were distributed normally and by Mann-Whitney $U$ test when the data were distributed non-normally. For categorical variables, statistical differences were evaluated by $\chi^{2}$ test. Significant relation between genes were assessed with Spearman correlation coefficient test in OriginPro 2017 (OriginLab Corporation, Northampton, Mass). Data were presented as mean $\pm \mathrm{SD}$ or $\mathrm{n}(\%) . P<.05$ was considered statistically significant. 

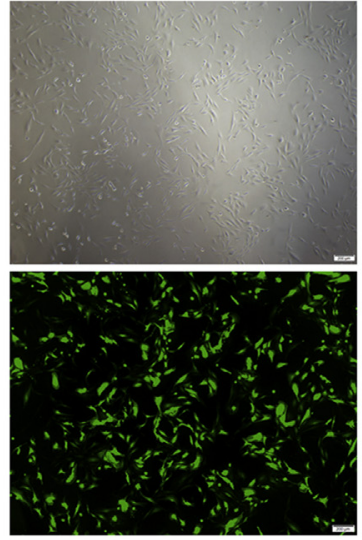

A

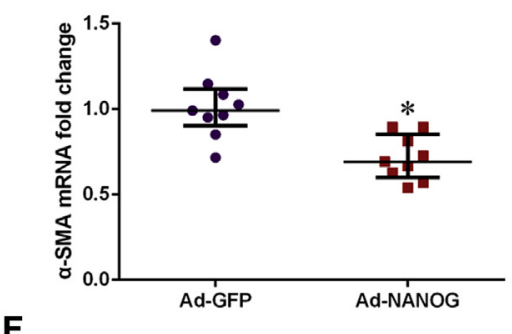

F

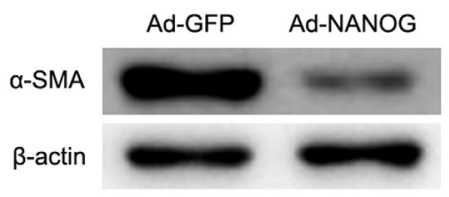

B
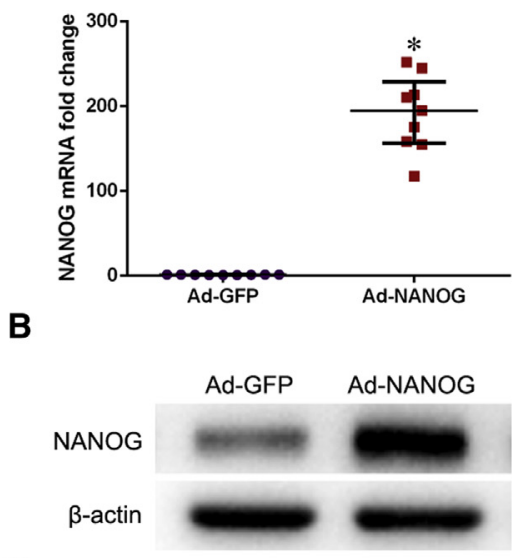

D

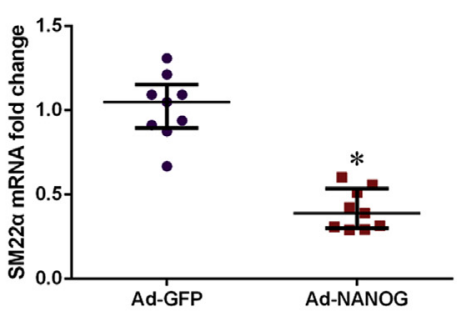

G

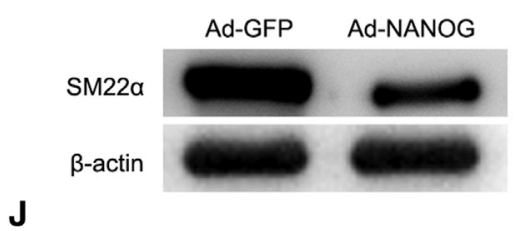

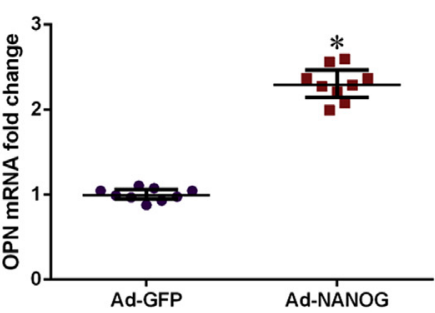

C

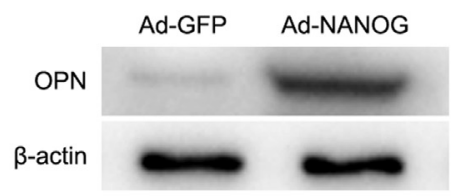

E

H
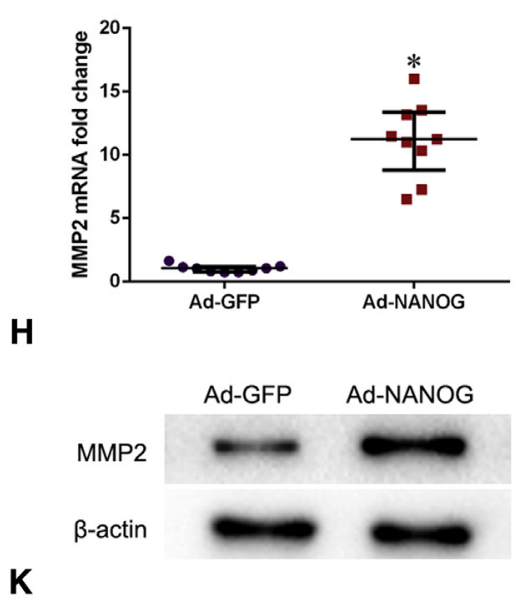

FIGURE 3. Overexpression of NANOG up-regulated the expression of OPN and VSMCs synthetic MMP2, whereas the expression of VSMCs contractile markers $\alpha$-SMA and SM22 $\alpha$ were down-regulated. A, Transfection efficiency of adenovirus vectors in VSMCs reached $\geq 90 \%(\times 40)$. B and D, Overexpression of NANOG in VSMCs transfected with Ad-NANOG with GFP tag was verified by qRT-PCR and Western blot on (B) mRNA and (D) protein levels, respectively. $\mathrm{C}$ and $\mathrm{E}$, The expression of $\mathrm{OPN}$ was significantly up-regulated in Ad-NANOG VSMCs both on (C) mRNA and (E) protein levels. F-H, VSMCs contractile markers (F) $\alpha$-SMA and (G) SM22 $\alpha$ were down-regulated whereas VSMCs synthetic marker (H) MMP2 was up-regulated in AdNANOG VSMCs on mRNA level. I-K, Protein level of VSMCs contractile markers (I) $\alpha$-SMA and (J) SM22 $\alpha$ was decreased and the expression of VSMCs synthetic marker (K) MMP2 was increased in Ad-NANOG VSMCs. $\beta$-actin was used as the internal reference in western blot. N $=9$ for qRT-PCR test and $\mathrm{N}=6$ for the other tests. VSMCs transfected with Ad-GFP served as a control. Error bars represent the median, 25\% and 75\% quartiles. The Student $t$ test was used to compare differences between 2 groups. $* P<.05$ versus Ad-GFP. NANOG, Nanog homeobox; Ad-GFP, green fluorescent protein control adenovirus; $A d$-NANOG, NANOG overexpression adenovirus; $O P N$, osteopontin; $\alpha$-SMA, $\alpha$-smooth muscle actin; SM22 $\alpha$, smooth muscle $22 \alpha$; $M M P 2$, marker matrix metalloproteinase 2 .

\section{RESULTS}

\section{TAD Aortic Media Expressed Greater NANOG and OPN Levels Accompanying VSMCs Phenotype \\ Switch}

We first collected TAD $(n=20)$ and controls $(n=10)$ aortic media specimens to examine the expression of NANOG. With qRT-PCR, Western blot, and immunohistochemistry assay, we found NANOG was highly expressed both in mRNA $(1.06 \pm 0.66$ vs $3.89 \pm 2.84, P<.05)$ and protein levels $(0.35 \pm 0.17$ vs $1.02 \pm 0.67, P<.05$ in Western and $1.67 \pm 0.82$ vs $14.67 \pm 2.42, P<.05$ in immunohistochemistry) in TAD aspecimens, which indicated that NANOG might be involved in the pathophysiologic processes of TAD (Figure 1, A, F, and $K)$. Then, we examined the expression level of OPN with the same methods and found OPN expression level also was greater $(2.88 \pm 2.06$ vs $14.04 \pm 14.71, P<.05$ in PCR; $0.38 \pm 0.14$ vs $1.20 \pm 0.90, P<.05$ in Western and $1.67 \pm 0.81$ vs $10.00 \pm 1.67, P<.05$ in immunohistochemistry) in TAD specimens (Figure $1, B, G$, and $L$ ). Last, we examined the expression level of VSMCs phenotype markers. We found contractile markers $\alpha$-SMA $(11.52 \pm 4.23$ vs $8.44 \pm 1.36, P<.05 ; 1.73 \pm 0.34$ vs $0.91 \pm 0.39, P<.05$, and $37.33 \pm 4.27$ vs $20.33 \pm 2.94$, $P<.05)$ and SM22 $\alpha(16.46 \pm 10.92$ vs $6.49 \pm 4.49$, $P<.05 ; 1.85 \pm 0.22$ vs $0.74 \pm 0.30, P<.05$, and 


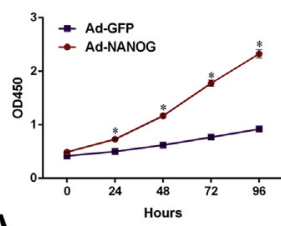

A

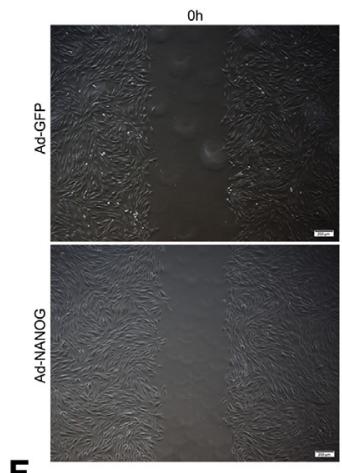

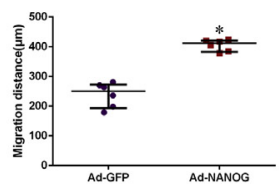

B

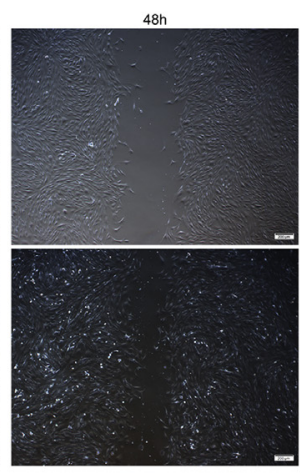

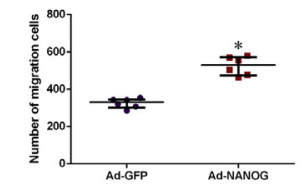

C

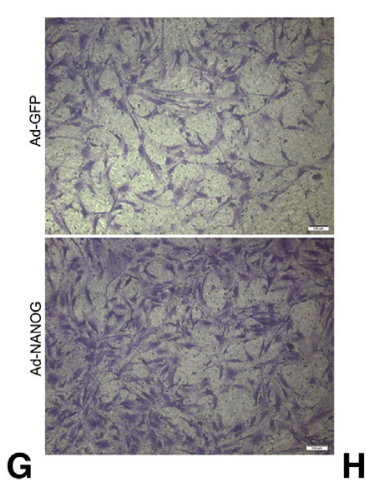

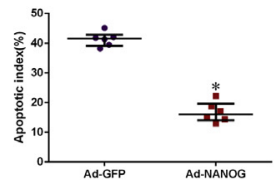

D

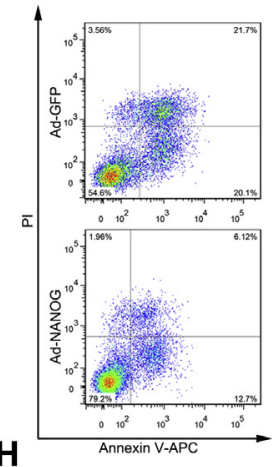

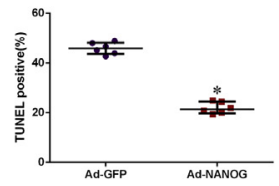

E

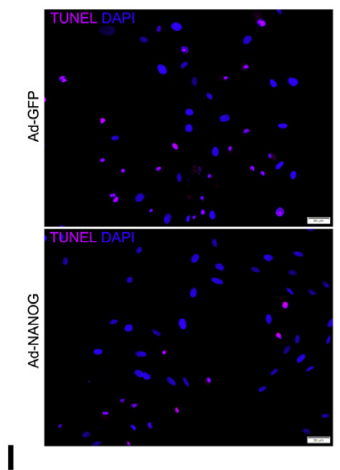

FIGURE 4. Overexpression of NANOG induced biological function change of VSMCs. A, CCK8 assay showed that VSMCs growth rate was significantly increased from 24 hours after transfected with Ad-NANOG with GFP tag. B and F, Scratch wound-healing assay showed the migration capability was enhanced in (F) Ad-NANOG VSMCs $(\times 40)$. B, The migration distance at 48 hours after scratch was quantified. C and G, Results of Transwell migration assay showed that the migration capability in vertical also was enhanced in (G), Ad-NANOG VSMCs $(\times 100)$. C, The number of migration cells 12 hours after plated was quantified. D and H, Apoptosis assay with flow cytometry showed apoptosis rate was decreased in Ad-NANOG VSMCs at 6 hours after ultraviolet exposure. E and I, TUNEL assay also showed apoptosis rate was decreased in Ad-NANOG VSMCs at 6 hours after ultraviolet exposure. N $=6$ for each test, VSMCs transfected with Ad-GFP served as a control. Error bars represent the median, 25\% and 75\% quartiles. The Student $t$ test was used to compare differences between 2 groups. ${ }^{*} P<.05$ versus Ad-GFP. Ad-GFP, Green fluorescent protein control adenovirus; $N A N O G$, Nanog homeobox; AdNANOG, NANOG overexpression adenovirus; TUNEL, terminal deoxynucleotidyl transferase dUTP nick end labeling; $P I$, propidium iodide; $D A P I, 4^{\prime}, 6$ diamidino-2-phenylindole.

$40.0 \pm 4.94$ vs $16.5 \pm 2.43, P<.05)$ were decreased and synthetic marker MMP2 $(0.21 \pm 0.30$ vs $2.28 \pm 1.97$, $P<.05 ; 0.57 \pm 0.23$ vs $1.48 \pm 0.83, P<.05$, and $2.67 \pm 1.03$ vs $12.50 \pm 2.07, P<.05)$ was increased in TAD specimens, which indicated that VSMCs switched from contractile to synthetic (Figure 1, C-E, $H-J$, and $M-O)$. Spearman correlation coefficient test showed the expression of NANOG was highly related with the expression of OPN (Figure E1, $K, \rho=0.959007$, $P<.05$ ) and MMP2 (Figure E1, $L, \rho=0.959398$, $P<.05)$. Semiquantitative analysis for Western and immunohistochemistry in this part was shown in Figure E1. All these results indicated that NANOG and OPN might be related with VSMCs phenotype switch in TAD.

\section{NANOG and OPN Were Highly Expressed in TAD VSMCs Accompanying Phenotype Switch in Vitro}

We isolated VSMCs from TAD and controls (Figure 2, $A$ ). First, to verify the purity of VSMCs, we detected the expression of $\alpha$-SMA and SM22 $\alpha$ by immunofluorescence and flow cytometry (Figure 2, $A$ and $B$ ), which were regarded as aortic VSMCs markers. ${ }^{8,28}$ Then, we examined the expression level of NANOG and OPN by qRT-PCR, Western blot, and immunofluorescence. The results showed both NANOG $(0.22 \pm 0.61$ vs $2.01 \pm 0.52, P<.05$ in mRNA and $0.74 \pm 0.10$ vs $1.36 \pm 0.11, P<.05$ in Western $)$ and OPN $(0.14 \pm 0.04$ vs $0.76 \pm 0.15, P<.05$ and $0.63 \pm 0.08$ vs $1.00 \pm 0.15$, $P<.05)$ were highly expressed in TAD VSMCs than controls (Figure 2, B, D, E, I, and $J$, and Figure E2, $C$ and $D)$. In addition, coimmunofluorescence staining of NANOG and OPN indicated that NANOG was distributed mainly in the nucleus and OPN was distributed mainly in the cytoplasm in TAD VSMCs (Figure 2, B). At last, we examined the expression level of $\alpha$-SMA (3.99 \pm 0.50 vs $0.19 \pm 0.04$, $P<.05$ in PCR and $0.82 \pm 0.07$ vs $0.46 \pm 0.07, P<.05$ in Western), SM22 $\alpha(3.95 \pm 0.69$ vs $0.86 \pm 0.28, P<.05$ and $1.01 \pm 0.10$ vs $0.59 \pm 0.10, P<.05)$ and MMP2 $(0.32 \pm 0.08$ vs $3.04 \pm 0.65, P<.05$ and $0.27 \pm 0.07$ vs $1.10 \pm 0.14, P<.05)$. The results confirmed the previous histologic detection results, which indicated TAD VSMCs switched from contractile to synthetic (Figure 2, $C, F-H$, and $K-M$, and Figure E2, $E-G)$. Coimmunofluorescence staining of NANOG and SM22 $\alpha$ showed high level NANOG accompanied with decreased expression of SM22 $\alpha$ in TAD VSMCs (Figure 2, $C$ ). The results in vitro confirmed that TAD VSMCs switched from contractile to synthetic; therefore, NANOG and OPN might play a role in this procedure. 

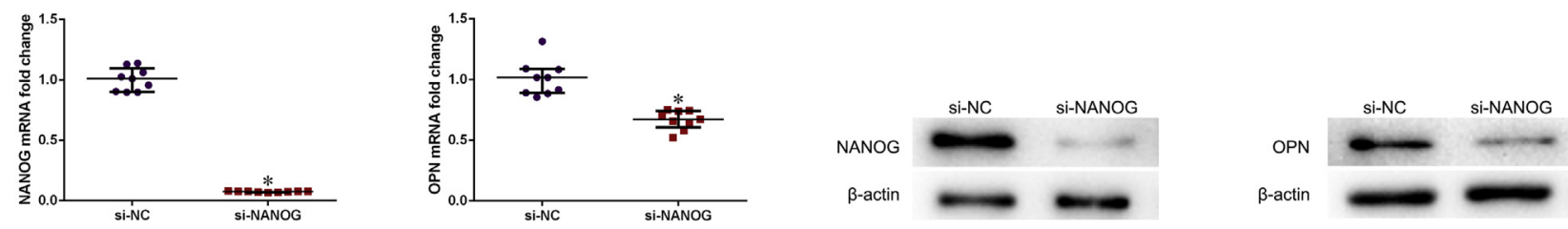

A

B

C

D

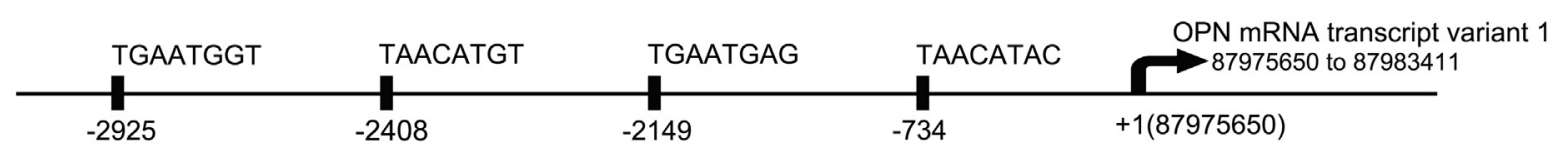

E

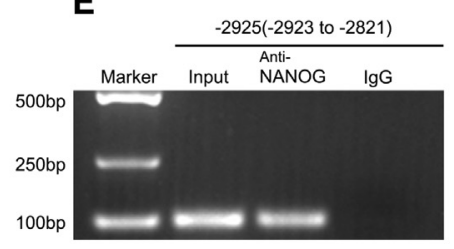

$\mathbf{F}$

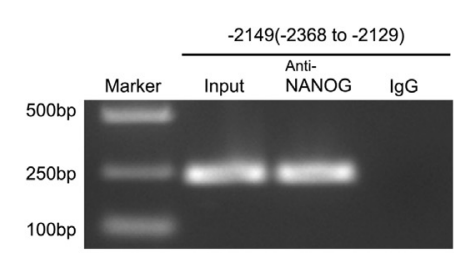

G

FIGURE 5. NANOG directly regulated the expression of OPN in VSMCs. A and C, Effective knockdown of NANOG in TAD VSMCs transfected with siNANOG was verified by qRT-PCR and Western blot on (A) mRNA and (C) protein levels, respectively. VSMCs transfected with scrambled si-NC served as a control. B and D, The expression of OPN was down-regulated both on (B) mRNA and (D) protein levels in si-NANOG VSMCs. E, Four predicted NANOG binding sites were in OPN promoter region. F and G, Chromatin immunoprecipitation of NANOG in VSMCs transfected with Ad-NANOG identified 2 functional binding sites, (F) -2925 and $(\mathrm{G})-2149$. Anti-RNA polymerase antibody served as positive control and normal mouse IgG served as negative control. $\beta$-actin was used as the internal reference in Western blot. $\mathrm{N}=9$ for qRT-PCR test and $\mathrm{N}=6$ for the other tests. Error bars represent the median, $25 \%$ and $75 \%$ quartiles. The Student $t$ test was used to compare differences between 2 groups. $* P<.05$ versus si-NC. NANOG, Nanog homeobox; si-NC, negative control siRNA; si-NANOG, NANOG-specific small interfering RNA; OPN, osteopontin.

\section{NANOG Overexpression Promoted the Expression of OPN and VSMCs Phenotype Switch}

VSMCs were isolated from control specimens and transfected with Ad-NANOG to investigate the effect of NANOG on the expression of OPN and VSMCs phenotype. Fluorescence observation, qRT-PCR, and Western blot were performed to detect transfection effects (Figure 3, A, $B$, and $D$ ). Relative NANOG protein level to $\beta$-actin was measured $(0.64 \pm 0.83$ vs $1.43 \pm 0.10, P<.05$, Figure E3, $A$ ) and it was close to that in TAD VSMCs. We first evaluated the related genes expression change on mRNA level compared with VSMCs transfected with Ad-green fluorescent protein. At 48 hours after transfection, the mRNA expression level of OPN increased to $2.30 \pm 0.20, P<.05$-fold in Ad-NANOG VSMCs (Figure 3,C). VSMCs synthetic markers MMP2 increased to $11.17 \pm 2.98, P<.05$-fold but VSMCs contractile markers $\alpha$-SMA and SM22 $\alpha$ decreased to $0.71 \pm 0.13$, $P<.05$ and $0.41 \pm 0.12, P<.05$-fold, respectively, in Ad-NANOG VSMCs (Figure $3, F-H$ ). Western blot results confirmed these changes on protein level (Figure 3, E, I-K, and Figure E3).

Then we evaluated the proliferation, migration, and apoptosis capabilities of VSMCs transfected with AdNANOG, which were considered as important functions of VSMCs in the pathogenesis of TAD. ${ }^{8,24,29}$ We used CCK8 assay to evaluate the proliferation function of
VSMCs. We found that VSMCs transfected with Ad-NANOG showed an enhanced proliferation function at 24, 48, 72, and 96 hours after transfection (Figure 4, A). Scratch wound-healing assay and Transwell migration assay were used to evaluate VSMCs migration function in lateral and vertical respectively. Results showed the migration function of Ad-NANOG VSMCs was increased both in lateral direction $(238.30 \pm 41.60$ vs $405.30 \pm 19.40 \mu \mathrm{m}, P<.05$, Figure $4, B$ and $F)$ and vertical direction $(325.70 \pm 25.88$ vs $524.9 \pm 49.66$ cells, $P<.05$, Figure $4, C$ and $G$ ) compared with Ad-GFP VSMCs. In apoptosis assay, Ad-NANOG VSMCs presented a significantly decreased apoptosis rate compared with AdGF $P$ VSMCs $(16.76 \% \pm 3.37 \%$ vs $41.37 \% \pm 2.38 \%$, $P<.05$ ) (Figure $4, D$ and $H$ ). TUNEL assay also showed an enhanced antiapoptosis capability in Ad-NANOG VSMCs $(21.89 \% \pm 2.27 \%$ vs $45.92 \% \pm 2.38 \%$, $P<.05$ ) (Figure 4, $E$ and $I$ ). These results illustrate that NANOG promoted the expression of OPN and VSMCs phenotype switch, which showed enhanced proliferation, migration, and antiapoptosis capabilities.

\section{NANOG Directly Regulated the Expression of OPN in VSMCs}

Because overexpression of NANOG could up-regulate the expression of OPN had been identified previously, we first evaluated whether interfere the expression of NANOG 


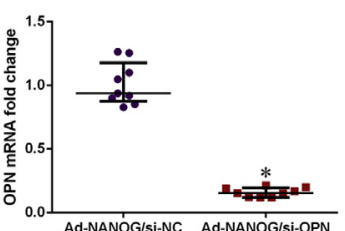

A

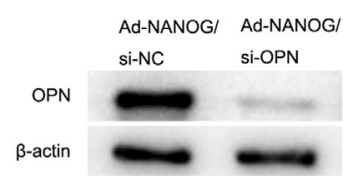

E

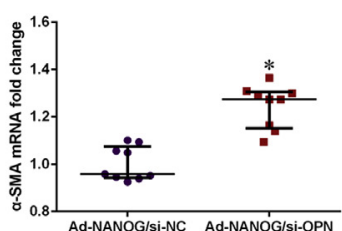

B

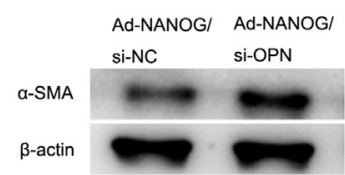

$\mathbf{F}$

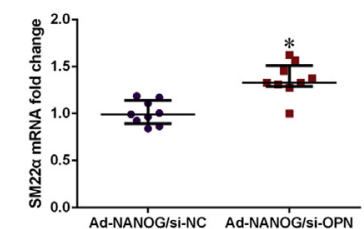

C

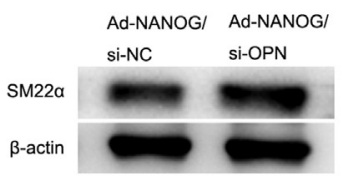

G

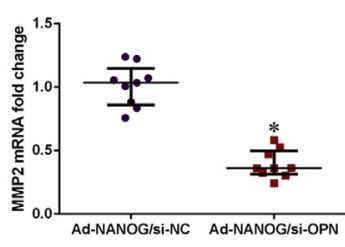

D

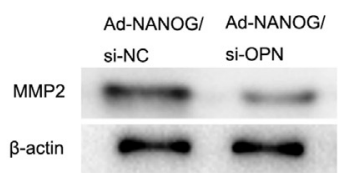

H

FIGURE 6. Down-regulation of OPN inhibited the function of NANOG on mRNA and protein levels. A and E, Effective knockdown of OPN in VSMCs cotransfected with NANOG overexpression adenovirus with green fluorescent protein tag and OPN-specific small interfering RNA adenovirus Ad-NANOG/ si-OPN was verified by quantitative real-time polymerase chain reaction and Western blot on (A) mRNA and (E) protein levels, respectively. B-D, The expression of VSMCs contractile markers (B) $\alpha$-SMA and (C) SM22 $\alpha$ were up-regulated and the expression of VSMCs synthetic marker (D) MMP2 was down-regulated in Ad-NANOG/si-OPN VSMCs on mRNA level. F-H, Western blot results showed changes of VSMC phenotype markers on protein level were similar to that on mRNA level. $\beta$-actin was used as the internal reference in Western blot. $\mathrm{N}=9$ for qRT-PCR test and $\mathrm{N}=6$ for Western blot. Ad-NANOG/si-NC served as a control. Error bars represent the median, $25 \%$ and $75 \%$ quartiles. The Student $t$ test and Mann-Whitney test were used to compare differences between 2 groups. ${ }^{*} P<.05$ versus Ad-NANOG/si-NC. OPN, Osteopontin; NANOG, Nanog homeobox; Ad-NANOG/si-NC, NANOG overexpression adenovirus/scrambled negative control siRNA adenovirus; Ad-NANOG/si-OPN, NANOG overexpression adenovirus/ OPN-specific small interfering RNA adenovirus; $\alpha$-SMA, $\alpha$-smooth muscle actin; SM22 $\alpha$, smooth muscle 22 $\alpha$; $M M P 2$, marker matrix metalloproteinase 2.

could down-regulate OPN. TAD VSMCs was used in NANOG interference experiment because VSMCs isolated from control specimens expressed low level of NANOG and OPN. The expression of NANOG was detected to evaluate the interference efficiency of si-NANOG (Figure 5, $A$ and $C)$. At 48 hours after transfection, mRNA level of OPN significantly decreased to $0.62 \pm 0.06, P<.05$ fold in si-NANOG VSMCs compared with controls (Figure 5, $B$ ). Western blot results also showed the expression of $\mathrm{OPN}$ was decreased in si-NANOG VSMCs $(1.13 \pm 0.13$ vs $0.52 \pm 0.04, P<.05$, Figure 5, $D$, and Figure E4, $H$ ). VSMCs phenotype markers also were detected, and results showed VSMC contractile markers $\alpha$-SMA and SM $22 \alpha$ increased to $2.18 \pm 0.27, P<.05$ and $2.85 \pm 0.29$, $P<.05$-fold, respectively, and VSMC synthetic marker MMP2 decreased to $0.31 \pm 0.07, P<.05$-fold in si-NANOG VSMCs (Figure E4, $A-C$ ). Western blot results confirmed these changes on a protein level (Figure E4, $D-F$ and $I-K)$.

Then, we investigated whether NANOG could bind directly to the promoter region of OPN. VSMCs isolated from controls transfected with Ad-NANOG were used in this part. We found there were 4 predicted NANOG binding sites in the promoter region of OPN (Figure 5,E). ChIP was used to identify which site was functional. The results showed 2 binding sites were functional, which were located at 2925bp (-2925) and 2149bp (-2149) upstream of the transcription start site of OPN (Figure 5, $F$ and $G$ ). Results in this part showed NANOG directly up-regulated OPN by binding to its promoter region in VSMCs.

\section{The Function of NANOG in VSMCs Was via OPN}

We evaluated whether the function of NANOG was via OPN in this part and VSMCs isolated from control specimens were used. The expression of OPN in Ad-NANOG VSMCs interfered with si-OPN (Figure 6, $A$ and $E$, and Figure E5, A). Ad-NANOG VSMCs transfected with si-NC served as a control. Compared with Ad-NANOG/ si-NC VSMCs, mRNA levels of $\alpha$-SMA, SM22 $\alpha$, and $\mathrm{MM} P 2$ were $1.24 \pm 0.09, P<.05,1.36 \pm 0.18, P<.05-$, and $0.39 \pm 0.11, P<.05$-fold 48 hours after transfection in Ad-NANOG/si-OPN VSMCs, respectively (Figure 6, $B-D)$. These changes were confirmed by Western blot on a protein level (Figure 6, F-H, and Figure E5, $B-D$ ).

Regarding the biological behaviors of VSMCs, CCK8 assay showed the proliferation capability of Ad-NANOG/ si-OPN VSMCs was decreased at 24, 48, 72, and 96 hours after transfection (Figure 7, A). The migration function of Ad-NANOG/si-OPN VSMCs also was decreased compared with Ad-NANOG/si-NC VSMCs $(207.5 \pm 22.47$ vs $494.9 \pm 32.86 \mu \mathrm{m}, P<.05$ in lateral and $204.00 \pm 34.72$ vs $372.80 \pm 26.01$ cells, $P<.05$ in vertical; Figure 7, $B, C, F$, and $G$ ). In addition, Ad-NANOG/si-OPN VSMCs showed a decreased apoptotic resistance capability compared with Ad-NANOG/si-NC VSMCs $(38.80 \pm 3.80 \%$ vs $22.61 \pm 2.60 \%, P<.05$ ) (Figure 7, $D$ and $H$ ). TUNEL assay also showed a decreased antiapoptosis capability in Ad-NANOG/si-OPN VSMCs $\quad(51.65 \% \quad \pm 2.17 \%$ vs $26.51 \% \pm 2.10 \%, P<.05$ ) (Figure $7, E$ and $I$ ). Taken together, the results showed interrupt the expression of OPN blocked the function of NANOG, which means the function of NANOG in VSMCs was through OPN. 


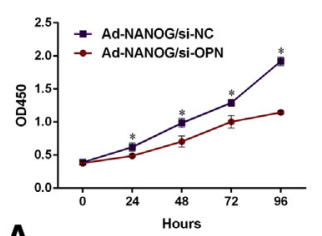

A

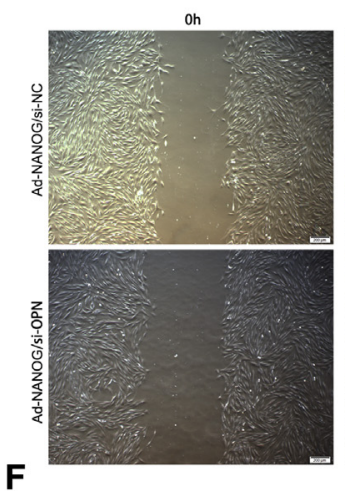

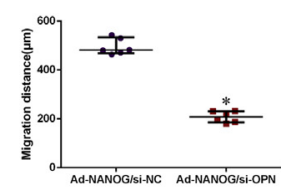

B

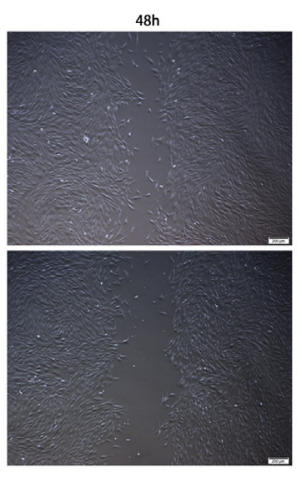

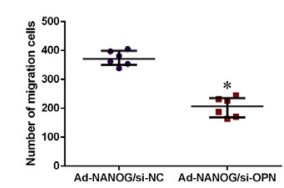

C

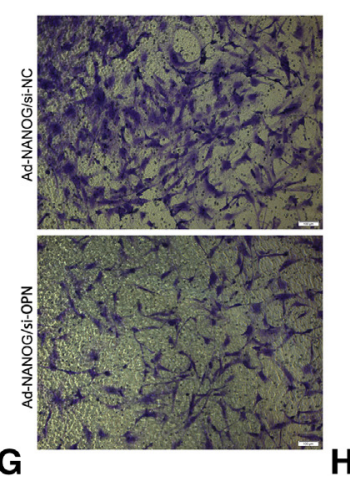

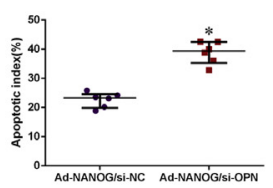

D

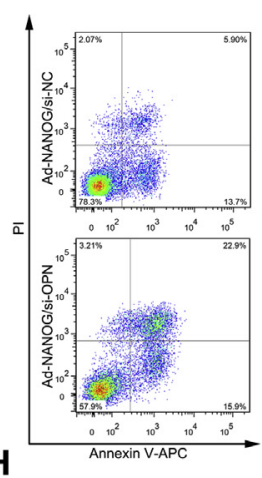

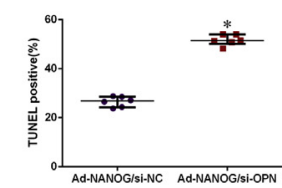

E

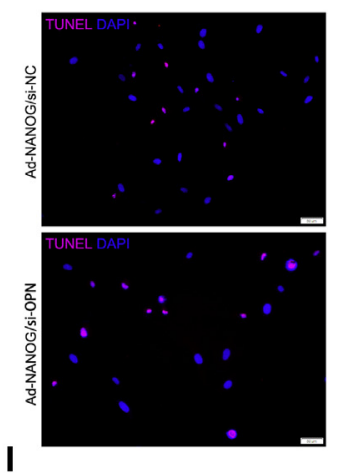

FIGURE 7. Down-regulation of OPN inhibited the effect of NANOG on VSMC biological functions. A, VSMCs growth rate was significantly decreased from 24 hours after being cotransfected with NANOG overexpression adenovirus with green fluorescent protein tag and Ad-NANOG/si-OPN. B and F, AdNANOG/si-OPN VSMCs showed $(F)$ decreased migration capability in scratch wound-healing assay $(\times 40)$. B, The migration distance at 48 hours after scratch was quantified. C and G, Results of Transwell migration assay showed that the migration capability in vertical (G) also was decreased in AdNANOG/si-OPN VSMCs $(\times 100)$. C, The number of migration cells 12 hours after plated was quantified. $\mathrm{D}$ and $\mathrm{H}$, The apoptosis rate was increased in Ad-NANOG/si-OPN VSMCs at 6 hours after ultraviolet exposure in apoptosis assay. E and I, TUNEL assay also showed apoptosis rate was increased in Ad-NANOG/si-OPN VSMCs at 6 hours after ultraviolet exposure. $\mathrm{N}=6$ for each test, Ad-NANOG/scrambled negative control siRNA adenovirus (Ad-NANOG/si-NC) served as a control. Error bars represent the median, 25\% and 75\% quartiles. The Student $t$ test was used to compare differences between 2 groups. $* P<.05$ versus Ad-NANOG/si-NC. OPN, Osteopontin; NANOG, Nanog homeobox; Ad-NANOG/si-NC, NANOG overexpression adenovirus/scrambled negative control siRNA adenovirus; Ad-NANOG/si-OPN, NANOG overexpression adenovirus/OPN-specific small interfering RNA adenovirus; TUNEL, terminal deoxynucleotidyl transferase dUTP nick end labeling; PI, propidium iodide.

\section{DISCUSSION}

In the present study, we first found NANOG was highly expressed in TAD aortic media and demonstrated that NANOG might play a role in the pathophysiologic processes of TAD via OPN. Our experiment results showed that by suppressing the expression of VSMCs contractile markers, up-regulating VSMC synthetic markers, and promoting VSMCs proliferation, migration, and antiapoptosis capacities, NANOG induced VSMCs phenotype switch via OPN.

VSMCs switching from the contractile to synthetic phenotype is an important biological behavior, which is marked with the enhanced proliferation, migration, antiapoptosis, up-regulation of synthetic markers, and down-regulation of contractile markers. ${ }^{9,13}$ Wang and colleagues $^{8}$ found VSMCs switched from contractile to synthetic type and that resulted in extracellular matrix degradation in TAD aortic wall. Our previous study also found VSMCs phenotype switch played a role in TAD. ${ }^{10,11}$ We used several detection methods and confirmed the overexpression of VSMCs synthetic marker MMP2 and the decreased expression of VSMCs contractile markers $\alpha$-SMA and SM22 $\alpha$ in TAD aortic wall compared with controls, both in vivo and in vitro.
These results illustrate that the VSMC phenotype switch may play a vital role in TAD.

To our knowledge, there are no studies on the participation of NANOG in aortic diseases. NANOG is an important transcription factor in mammalian embryo development and is essential for maintaining pluripotency and self-renewal of embryonic stem cells. ${ }^{19}$ NANOG is associated with cell differentiation and is highly expressed in embryonic and undifferentiated cells but lowly expressed in somatic and differentiated cells. ${ }^{30}$ Because of these characteristics, NANOG is important for the dedifferentiation process of somatic cells, like tumor, and induced pluripotency. In a previous study, downregulation of NANOG suppressed the proliferation and migration of breast cancer cells. ${ }^{31}$ Arif and colleagues ${ }^{32}$ found NANOG enhanced the antiapoptosis capacity in tamoxifen-resistant breast cancer cells. Moreover, VSMCs phenotype switch is regarded as a dedifferentiation process, ${ }^{21}$ and our experiment results showed NANOG participated in this process.

In this study, we found NANOG was highly expressed in TAD aortic specimens. In VSMCs, high-level NANOG promoted the expression of synthetic marker MMP2 and reduced the level of contractile markers $\alpha$-SMA and 
SM22 $\alpha$. Meanwhile, NANOG silence inhibited these changes in TAD VSMCs. In addition, NANOG also induced alterations in VSMCs function and was involved in increased proliferation, migration, and antiapoptosis capacities. MMP2 degrades elastic fibers in extracellular matrix. ${ }^{10} \alpha$-SMA is an important protein to maintain the morphology and contractibility of VSMCs. ${ }^{5}$ In contrast, although SM22 $\alpha$ is an important component of cytoskeleton, it is not indispensable for the contractibility of VSMCs. ${ }^{33}$ The overexpression of MMP2 and reduced level of $\alpha$-SMA and SM22 $\alpha$ impair the elastin-contractile units of VSMCs. ${ }^{5,34}$ All these changes are important promoting factors for TAD. 5,35

We considered how NANOG performed aforementioned functions in VSMCs and found OPN was the downstream gene of NANOG in human embryonic stem cells. ${ }^{22}$ It was reported the expression of OPN also was increased in TAD aortic specimens and overexpression of OPN could transform VSMCs from the contractile to synthetic phenotype. ${ }^{8,14}$ Through our experiments, we found OPN was indispensable in carrying out the functions of NANOG in VSMCs. To verify whether NANOG promoted VSMCs phenotype switch through OPN, we interfered OPN in Ad-NANOG VSMCs and found it suppressed the phenotype switch induced by NANOG. That meant OPN was not only high expressed in TAD but also participates in the pathophysiologic processes of it by promoting VSMCs phenotype switch.

The regulatory relationship between NANOG and OPN in VSMCs has not been investigated. Kunarso and colleagues $^{22}$ found NANOG bound to the promoter region of OPN in human embryonic stem cells by ChIP sequencing assay. We first found up- and down-regulation of NANOG could induce corresponding changes in OPN, which indicated the expression of OPN was regulated by NANOG. To further investigate the relationship between NANOG and OPN, we used ChIP to verify whether NANOG directly regulate OPN in VSMCs. Our results showed that NANOG directly promoted the expression of OPN by binding to its promoter region in VSMCs.

In conclusion, our study has shown aortic media specimens from patients with TAD presented high-level NANOG compared with controls. NANOG induced VSMCs phenotype switch from contractile to synthetic type in vitro. Our further investigations showed the function of NANOG was via OPN and NANOG directly upregulated OPN by binding to its promoter region. These findings suggest that NANOG is highly expressed in TAD and promotes the VSMCs phenotype switch by directly up-regulating OPN.

\section{Limitations}

Our study has some limitations. First, the function of NANOG was investigated in vitro, which did not complete simulate in vivo environment. The present findings just indicated NANOG was associated with TAD, and there were no cause and effect between NANOG and TAD. To further investigate the association between NANOG and TAD, an in vivo animal experiment is needed. Second, our study found NANOG was highly expressed in TAD VSMCs and its function was via OPN. But the reason of NANOG overexpression in the pathogenesis of TAD is still unclear. Further research is needed to fully illustrate the regulatory mechanism of NANOG in TAD. Third, we investigated the role of NANOG in TAD. However, the relationship between NANOG and other genes that may result in TAD remains to be studied. ${ }^{3,11,24}$ Fourth, we focused on the aortic media and VSMCs, aortic endothelial and adventitial cells were not included in the present study. We believe aortic endothelial and adventitial cells also play a role in TAD and need to be further investigated. Finally, this study was limited by the small sample size, and the controls in this study also had cardiovascular diseases, which might induce biases. The findings in the present study need to be further confirmed.

\section{Conflict of Interest Statement}

Authors have nothing to disclose with regard to commercial support.

\section{References}

1. Zhu JM, Qi RD, Chen L, Liu W, Li CN, Fan ZM, et al. Surgery for acute type A dissection using total arch replacement combined with stented elephant trunk implantation: preservation of autologous brachiocephalic vessels. J Thorac Cardiovasc Surg. 2015;150:101-5.

2. Chen LW, Wu XJ, Dai XF, Liao DS, Li C, Wang QM, et al. A self-adaptive triple-branched stent graft for arch repair during open type A dissection surgery. J Thorac Cardiovasc Surg. 2015;149:1278-83.e1271.

3. Kimura T, Shiraishi K, Furusho A, Ito S, Hirakata S, Nishida N, et al. Tenascin C protects aorta from acute dissection in mice. Sci Rep. 2014;4:4051.

4. Wang X, LeMaire SA, Chen L, Shen YH, Gan Y, Bartsch H, et al. Increased collagen deposition and elevated expression of connective tissue growth factor in human thoracic aortic dissection. Circulation. 2006;114:I200-5.

5. Karimi A, Milewicz DM. Structure of the elastin-contractile units in the thoracic aorta and how genes that cause thoracic aortic aneurysms and dissections disrupt this structure. Can J Cardiol. 2016;32:26-34.

6. Owens GK, Kumar MS, Wamhoff BR. Molecular regulation of vascular smooth muscle cell differentiation in development and disease. Physiol Rev. 2004;84: 767-801.

7. Kawai-Kowase K, Owens GK. Multiple repressor pathways contribute to phenotypic switching of vascular smooth muscle cells. Am J Physiol Cell Physiol. 2007;292:C59-69.

8. Wang L, Zhang J, Fu W, Guo D, Jiang J, Wang Y. Association of smooth muscle cell phenotypes with extracellular matrix disorders in thoracic aortic dissection. J Vasc Surg. 2012;56:1698-709. 1709.e1691.

9. Zhu SB, Zhu J, Zhou ZZ, Xi EP, Wang RP, Zhang Y. TGF-betal induces human aortic vascular smooth muscle cell phenotype switch through PI3K/AKT/ID2 signaling. Am J Transl Res. 2015;7:2764-74.

10. Yuan Y, Wang C, Xu J, Tao J, Xu Z, Huang S. BRG1 overexpression in smooth muscle cells promotes the development of thoracic aortic dissection. BMC Cardiovasc Disord. 2014;14:144.

11. Yan Y, Tan MW, Xue X, Ding XY, Wang GK, Xu ZY. Involvement of Oct4 in the pathogenesis of thoracic aortic dissection via inducing the dedifferentiated phenotype of human aortic smooth muscle cells by directly upregulating KLF5. J Thorac Cardiovasc Surg. 2016;152:820-9. 
12. Liu J, Ren Y, Kang L, Zhang L. Oxidized low-density lipoprotein increases the proliferation and migration of human coronary artery smooth muscle cells through the upregulation of osteopontin. Int J Mol Med. 2014;33:1341-7.

13. Weintraub AS, Schnapp LM, Lin X, Taubman MB. Osteopontin deficiency in rat vascular smooth muscle cells is associated with an inability to adhere to collagen and increased apoptosis. Lab Invest. 2000;80:1603-15.

14. Gao H, Steffen MC, Ramos KS. Osteopontin regulates alpha-smooth muscle actin and calponin in vascular smooth muscle cells. Cell Biol Int. 2012;36: 155-61.

15. Yan W, Qian C, Zhao P, Zhang J, Shi L, Qian J, et al. Expression pattern of osteopontin splice variants and its functions on cell apoptosis and invasion in glioma cells. Neuro Oncol. 2010;12:765-75.

16. Seo KW, Lee SJ, Ye BH, Kim YW, Bae SS, Kim CD. Mechanical stretch enhances the expression and activity of osteopontin and MMP-2 via the Akt1/AP-1 pathways in VSMC. J Mol Cell Cardiol. 2015;85:13-24.

17. Cole MF, Johnstone SE, Newman JJ, Kagey MH, Young RA. Tcf3 is an integral component of the core regulatory circuitry of embryonic stem cells. Genes Dev. 2008;22:746-55.

18. Mitsui K, Tokuzawa Y, Itoh H, Segawa K, Murakami M, Takahashi K, et al. The homeoprotein Nanog is required for maintenance of pluripotency in mouse epiblast and ES cells. Cell. 2003;113:631-42.

19. Silva J, Nichols J, Theunissen TW, Guo G, van Oosten AL, Barrandon O, et al. Nanog is the gateway to the pluripotent ground state. Cell. 2009;138:722-37.

20. Hyslop L, Stojkovic M, Armstrong L, Walter T, Stojkovic P, Przyborski S, et al. Downregulation of NANOG induces differentiation of human embryonic stem cells to extraembryonic lineages. Stem Cells. 2005;23:1035-43.

21. Frismantiene A, Dasen B, Pfaff D, Erne P, Resink TJ, Philippova M, T-cadherin promotes vascular smooth muscle cell dedifferentiation via a GSK3beta-inactivation dependent mechanism. Cell Signal. 2016;28: 516-30.

22. Kunarso G, Chia NY, Jeyakani J, Hwang C, Lu X, Chan YS, et al. Transposable elements have rewired the core regulatory network of human embryonic stem cells. Nat Genet. 2010;42:631-4.

23. Zhang XW, Zhang BY, Wang SW, Gong DJ, Han L, Xu ZY, et al. Twist-related protein 1 negatively regulated osteoblastic transdifferentiation of human aortic valve interstitial cells by directly inhibiting runt-related transcription factor 2 . J Thorac Cardiovasc Surg. 2014;148:1700-8.e1701.

24. Liao WL, Tan MW, Yuan Y, Wang GK, Wang C, Tang H, et al. Brahma-related gene 1 inhibits proliferation and migration of human aortic smooth muscle cells by directly up-regulating Ras-related associated with diabetes in the pathophysiologic processes of aortic dissection. J Thorac Cardiovasc Surg. 2015;150:1292-301.e1292.

25. Zhang M, Liu X, Zhang X, Song Z, Han L, He Y, et al. MicroRNA-30b is a multifunctional regulator of aortic valve interstitial cells. J Thorac Cardiovasc Surg. 2014;147:1073-80.e1072.

26. Wang S, Zhang X, Yuan Y, Tan M, Zhang L, Xue X, et al. BRG1 expression is increased in thoracic aortic aneurysms and regulates proliferation and apoptosis of vascular smooth muscle cells through the long non-coding RNA HIF1A-AS 1 in vitro. Eur J Cardiothorac Surg. 2015;47:439-46.

27. Shi X, Guo LW, Seedial SM, Si Y, Wang B, Takayama T, et al. TGF-beta/Smad3 inhibit vascular smooth muscle cell apoptosis through an autocrine signaling mechanism involving VEGF-A. Cell Death Dis. 2014:5:e1317.

28. Solway J, Seltzer J, Samaha FF, Kim S, Alger LE, Niu Q, et al. Structure and expression of a smooth muscle cell-specific gene, SM22 alpha. J Biol Chem. 1995;270:13460-9

29. Shen YH, Zhang L, Ren P, Nguyen MT, Zou S, Wu D, et al. AKT2 confer protection against aortic aneurysms and dissections. Circ Res. 2013;112:618-32.

30. Hart AH, Hartley L, Ibrahim M, Robb L. Identification, cloning and expression analysis of the pluripotency promoting Nanog genes in mouse and human. Dev Dyn. 2004:230:187-98.

31. Han ML, Wang F, Gu YT, Pei XH, Ge X, Guo GC, et al. MicroR-760 suppresses cancer stem cell subpopulation and breast cancer cell proliferation and metastasis: by down-regulating NANOG. Biomed Pharmacother. 2016;80: 304-10.

32. Arif K, Hussain I, Rea C, El-Sheemy M. The role of Nanog expression in tamoxifen-resistant breast cancer cells. Onco Targets Ther. 2015;8:1327-34.

33. Feil S, Hofmann F, Feil R. SM22alpha modulates vascular smooth muscle cel phenotype during atherogenesis. Circ Res. 2004;94:863-5

34. Borges LF, Touat Z, Leclercq A, Zen AA, Jondeau G, Franc B, et al. Tissue diffusion and retention of metalloproteinases in ascending aortic aneurysms and dissections. Human Pathol. 2009;40:306-13.

35. Goldfinger JZ, Halperin JL, Marin ML, Stewart AS, Eagle KA, Fuster V Thoracic aortic aneurysm and dissection. J Am Coll Cardiol. 2014;64 1725-39.

Key Words: aortic dissection, NANOG, OPN, smooth muscle cell, phenotype switch 


\section{APPENDiX E1. CELl CULTURE}

We isolated vascular smooth muscle cells (VSMCs) from 6 patients with thoracic aortic dissection and 6 controls. To ensure the purity of VSMCs, aortic intima and adventitia were stripped from the specimens, the rest were cut into 4- $\mathrm{mm}^{2}$ tissue blocks, and each block was adhered in a 24-well culture plate. The plate was inversed and incubated at $37^{\circ} \mathrm{C}$ with $5.0 \%$ carbon dioxide for 1 hour, then $200 \mu \mathrm{L}$ Medium 231 with smooth muscle growth supplements (Cascade Biologics, Waltham, Mass) was added into each well. After that, the plate was incubated at $37^{\circ} \mathrm{C}$ with $5.0 \%$ carbon dioxide and the culture medium was replaced every 72 hours. When cells grew to confluence, tissue blocks were transferred to another plate for reprimary culture and cells were passaged. Immunofluorescence for VSMCs markers $\alpha$-smooth muscle actin ( $\alpha$-SMA) and smooth muscle $22 \alpha$ (SM22 $\alpha$ ) and flow cytometry (Miltenyi Biotec, Teterow, Germany) for VSMCs marker SM22 $\alpha$ were used for the identification of VSMCs. VSMCs from passage 3 to passage 8 were used for experiments in this study.

\section{Quantitative Real-Time Polymerase Chain Reaction (qRT-PCR)}

To reduce the interference from endothelial cells and myofibroblasts, tissue specimens used for qRT-PCR were stripped the intima and adventitia, following our previous description. ${ }^{11,24}$ The procedure of total RNA extraction and cDNA synthesis was the same with our previous description. ${ }^{25}$ LightCycler 480 Real-Time PCR System (F. Hoffmann-La Roche AG, Basel, Switzerland) with SYBR Premix EX Taq (TaKaRa Bio, Dalian, China) were used for qRT-PCR. Comparative computed tomography method was used for result analysis. The primers for each gene were as follows: NANOG forward: 5'-GCCGAA GAATAGCAATGGT-3', NANOG reverse: 5'-CCAGG TCTGAGTGTTCCA-3'; OPN forward: $5^{\prime}$-TGATGGCCG AGGTGATAGTG-3', OPN reverse: 5'-CCAATCAGAA GGCGCGTT-3'; $\alpha$-SMA forward: 5'-GGCGGTGCTGTC TCTCTATG- $3^{\prime}, \alpha$-SMA reverse: $5^{\prime}$-GGAATAGCCACG CTCAGTCA-3'; SM22 $\alpha$ forward: 5'-CCAGACATGGC CAACAAGG- $3^{\prime}$, SM2 $2 \alpha$ reverse: $5^{\prime}$-CGCCATTCTTCAG CCAGAC-3'; MMP2 forward: 5'-GAATGAAGCACAGCA GGTCTC-3', MMP2 reverse: 5'-TGAAGCCAAGCGGTC TAAGT-3'; GAPDH forward: 5'-ACCACCATGGAGAAG GCTG-3', GAPDH reverse: 5'-GGTCATGAGTCCTTC CACGA- $3^{\prime}$.

\section{Terminal Deoxynucleotidyl Transferase dUTP Nick End Labeling (TUNEL) Assay}

One-Step TUNEL Apoptosis Assay Kit (KeyGEN BioTECH, Nanjing, China) was used for the TUNEL assay.
In brief, VSMCs were fixed with $4 \%$ paraformaldehyde for 15 minutes and then incubated with $0.1 \%$ Triton X-100 for 15 minutes. After that, VSMCs were treated with the mixture of terminal deoxynucleotidyl transferase enzyme and biotin-11-dUTP for 1 hour and then incubated with fluorescent labeling buffer for 30 minutes. The apoptotic cells were stained with red nuclei and 4',6-diamidino-2phenylindole staining was used to count total cell number.

\section{Immunofluorescence}

VSMCs were seeded on coverslips in a 24-well plate. VSMCs were rinsed with phosphate-buffered saline, $75 \%$ ethanol was used for fixation, and $0.5 \%$ Triton $\mathrm{X}-100$ was used for membrane penetration. Then cells were blocked with $5 \%$ goat serum for 30 minutes and treated with primary antibodies overnight at $4^{\circ} \mathrm{C}$. Finally, VSMCs were treated with secondary antibodies for 1 hour to visualize the proteins. DAPI was used for nucleus counterstain. Primary antibodies used were anti-NANOG (4903S; Cell Signaling Technology, Boston, Mass), antiNANOG (sc-293121; Santa Cruz Biotechnology, Santa Cruz, Calif), anti-OPN (BS1264; Bioworld Technology, Minneapolis, Minn), anti-OPN (sc-21742; Santa Cruz Biotechnology), anti- $\alpha$-SMA (BM0002; Boster Technology, Wuhan, China) and anti-SM22 $\alpha$ (ab14106; Abcam, Cambridge, UK). The secondary antibodies were from Jackson ImmunoResearch (111-585-003 and 115-545003, West Grove, Pa).

\section{Chromatin Immunoprecipitation (ChIP)}

EpiQuik ChIP Kit (EpiGentek, Farmingdale, NY) was used for ChIP assay following the manufacturer's instructions. VSMCs transfected with Ad-NANOG for 48 hours and anti-NANOG antibody (ChIP grade, 5232S, Cell Signaling Technology, Boston, Mass) were used in this part. Anti-RNA polymerase antibody was used as positive control and normal mouse IgG was used as negative control. VCX400 ultrasonic processor (Sonics \& Materials, Newtown, Conn) with the recommended shearing conditions was used for DNA sonication. NANOG binding sites in the promoter region of OPN was predicted by MatInspector (Genomatix software, Munich, Germany). The primers of the binding sites in OPN promoter area were as follows: -734 forward: 5'-ATAGTCCTTAAGATA CGCAGAGCAT- $3^{\prime}$, reverse: $5^{\prime}$-CCAGCCTACCTATCCTT GTTCC-3'; -2149 forward: 5'-ACAACCAGCAGCAGC AT-3', reverse: 5'-CCGACTTCTACACTCATTCAA-3'; - 2408 forward: $5^{\prime}$-CAACCAATCATTAGCCAATC-3', reverse: 5'-GATGCTGCGTCATTCTG-3'; -2925 forward: 5'-GATG AATGGTGCTAGGCTTCC-3' ${ }^{\prime}$, reverse: $5^{\prime}$-TTGAGTCAA GGTTCAGACATGA-3'. 

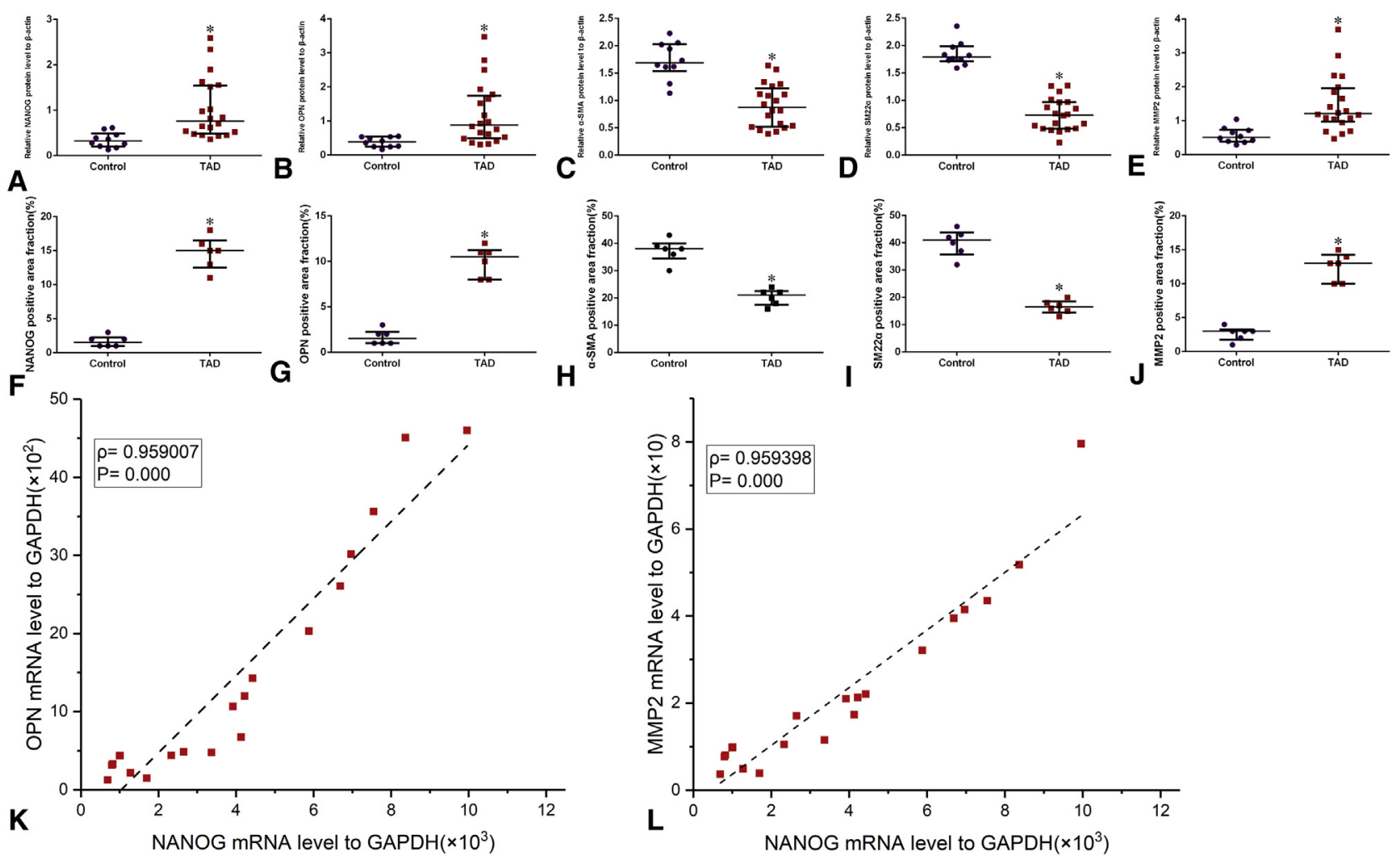

FIGURE E1. Semiquantitative analysis for Western blot and immunohistochemical results in Figure 1 and Pearson correlation coefficient test for NANOG, OPN, and MMP2 in patients with TAD. A-E, Western blot semiquantitative results showed protein levels of (A) NANOG; (B) OPN; and (E) MMP2 were greater whereas (C) $\alpha$-SMA and (D) SM22 $\alpha$ were lower in the TAD group $(\mathrm{n}=20)$ compared with the control group $(\mathrm{n}=10)$. F-J, Immunohistochemistry semiquantitative results showed enhanced staining of (F) NANOG; (G) OPN; and (J) MMP2 and decreased staining of (H) $\alpha$-SMA and (I) SM22 $\alpha$ in the TAD group $(n=6)$ compared with the control group $(n=6) . K$ and $L$, Pearson correlation coefficient test showed the strong positive correlation between NANOG and (K) OPN/L, MMP2 on mRNA level in patients with TAD $(n=20)$. Error bars represent the median, $25 \%$ and $75 \%$ quartiles. Positive area fraction meant the fraction of positive signal in the view field. Student $t$ test and Mann-Whitney $U$ test were used to compare differences between 2 groups. Spearman correlation coefficient test was used to assess the significant relation between genes. $* P<.05$ versus control. NANOG, Nanog homeobox; TAD, thoracic aortic dissection; $O P N$, osteopontin; $\alpha$-SMA, $\alpha$-smooth muscle actin; SM22 $\alpha$, smooth muscle 22 $\alpha$; MMP2, matrix metalloproteinase 2; GAPDH, glyceraldehyde-3-phosphate dehydrogenase. 

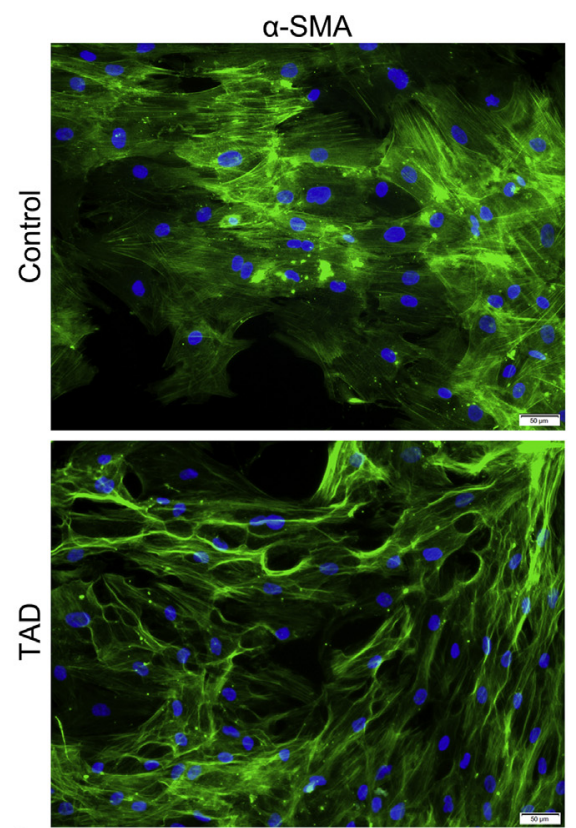

A
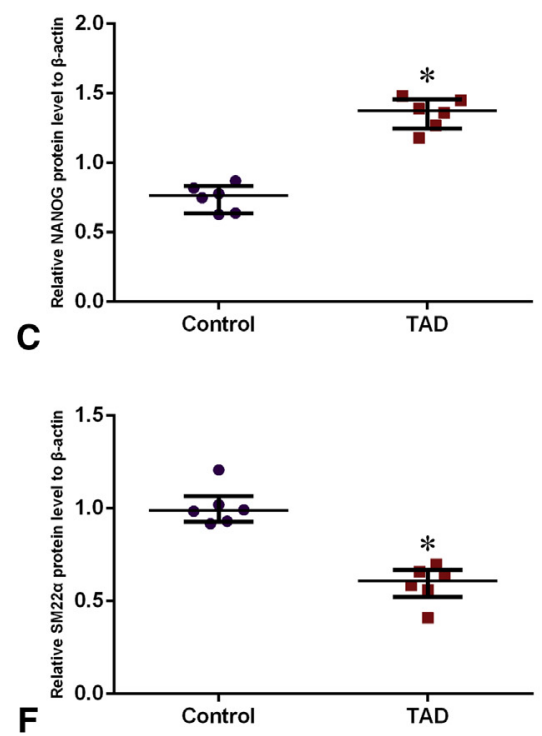

SM22 $\alpha$
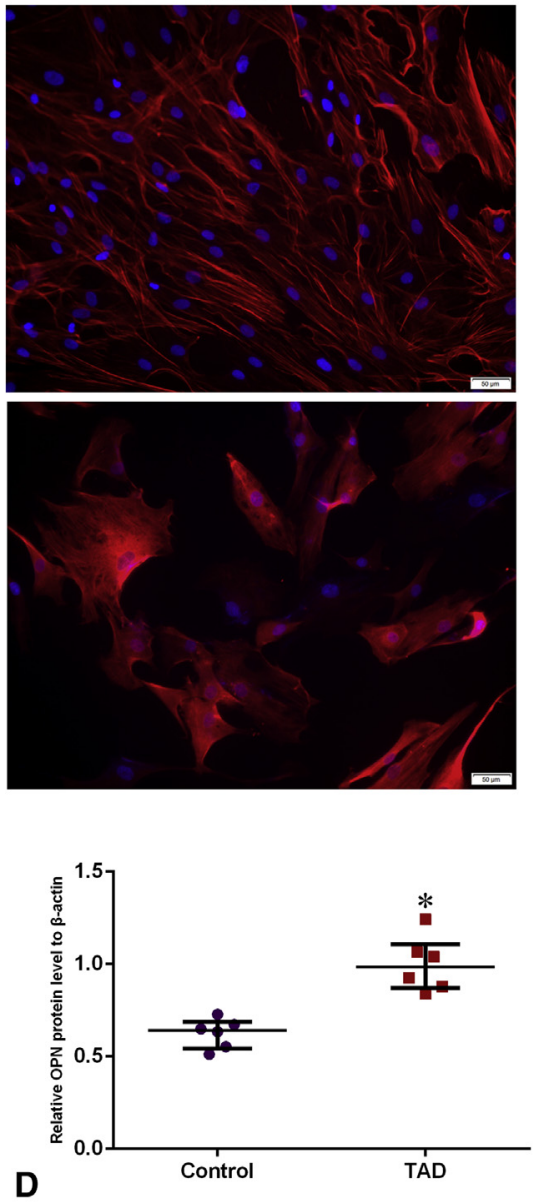

B
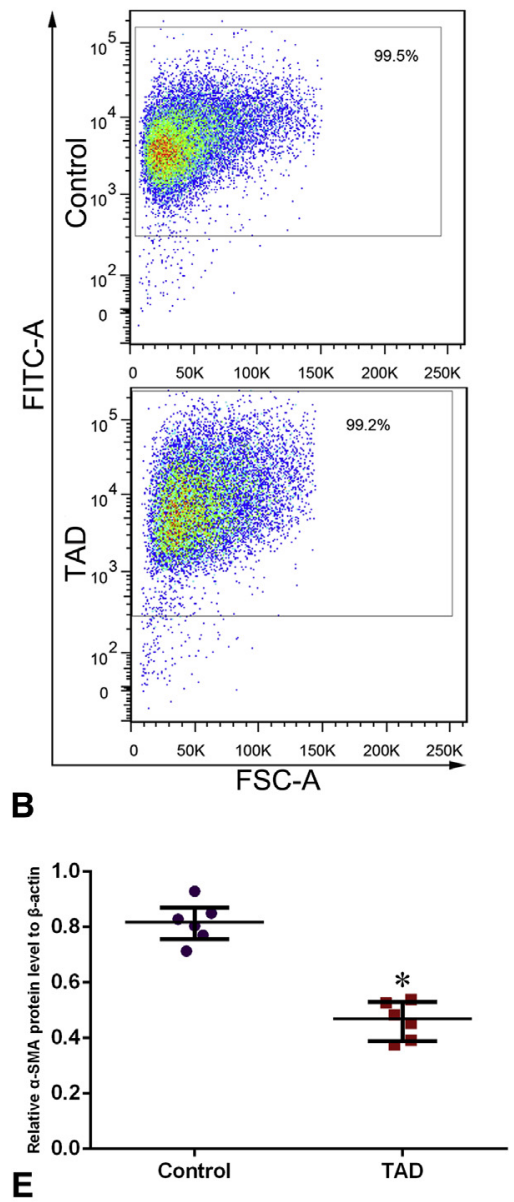

FIGURE E2. Identification of VSMCs and semiquantitative analysis for Western blot results in Figure 2. A, Immunofluorescent results showed VSMCs markers $\alpha$-SMA (green) and SM22 $\alpha$ (red) was expressed in almost all cells isolated from TAD and control patients $(\times 200)$. The nucleus was stained as blue with DAPI. B, Flow cytometry showed that the SM22 $\alpha$-positive rate was $\geq 99 \%$ in VSMCs isolated from TAD and control patients. C-G, Semiquantitative analysis for Western blot results in Figure $2(\mathrm{n}=6)$. Error bars represent the median, $25 \%$ and $75 \%$ quartiles. Student $t$ test was used to compare differences between 2 groups. $* P<.05$ versus control. $\alpha$-SMA, $\alpha$-smooth muscle actin; SM22 $\alpha$, smooth muscle $22 \alpha$; FITC-A, fluorescein isothiocyanate area; TAD, thoracic aortic dissection; FSC-A, forward scatter area; $N A N O G$, Nanog homeobox; OPN, osteopontin; MMP2, matrix metalloproteinase 2. 

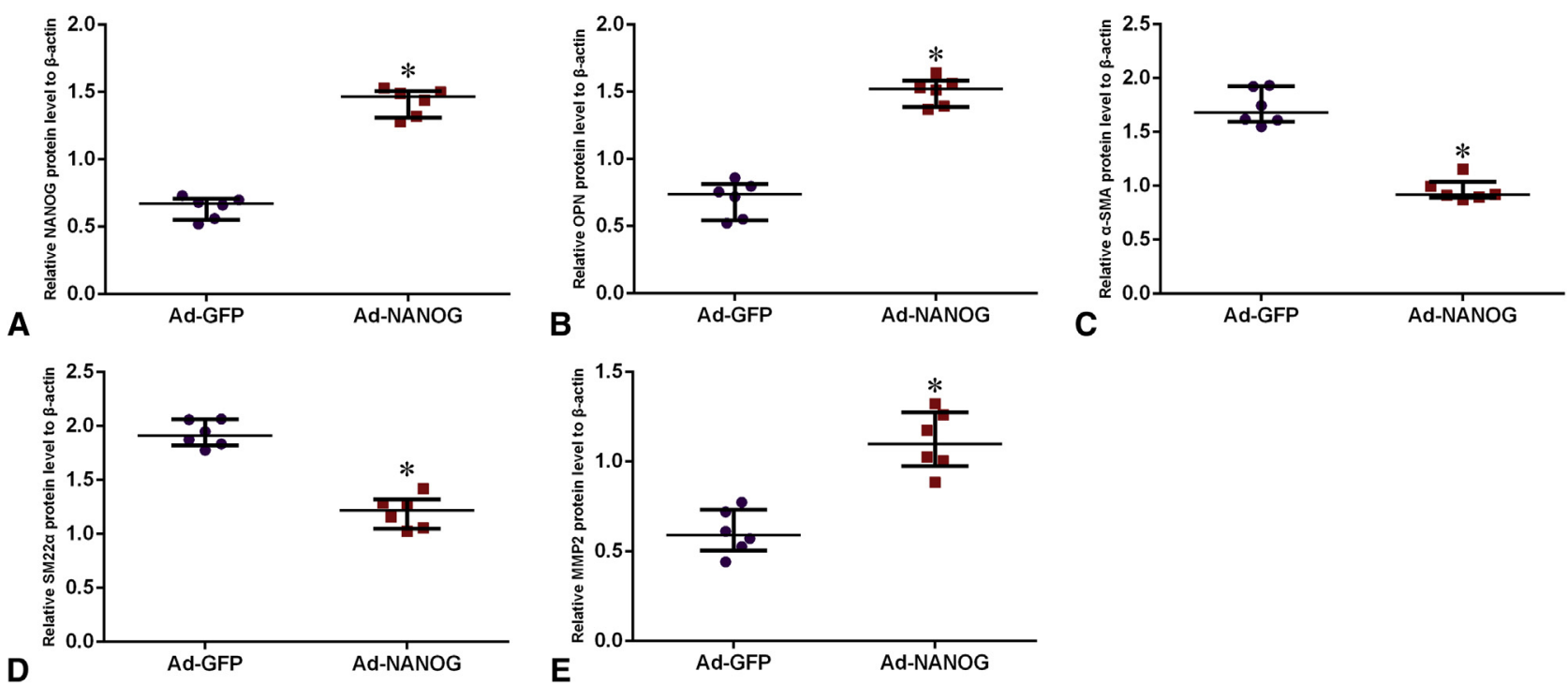

FIGURE E3. Semiquantitative analysis for Western blot results in Figure 3. Western blot semiquantitative results showed protein levels of (A) NANOG; (B) OPN; and (E) MMP2 were greater, whereas (C) $\alpha$-SMA and (D) SM22 $\alpha$ were lower in VSMCs transfected with Ad-NANOG (n = 6) compared with VSMCs transfected with Ad-GFP $(\mathrm{n}=6)$. Error bars represent the median, $25 \%$ and $75 \%$ quartiles. Student $t$ test was used to compare differences between 2 groups. ${ }^{*} P<.05$ versus Ad-GFP. NANOG, Nanog homeobox; Ad-GFP, green fluorescent protein control adenovirus; Ad-NANOG, NANOG overexpression adenovirus; $O P N$, osteopontin; $\alpha$-SMA, $\alpha$-smooth muscle actin; $S M 22 \alpha$, smooth muscle 22 $\alpha$; MMP2, matrix metalloproteinase 2. 


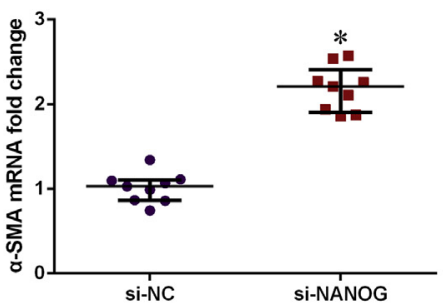

A

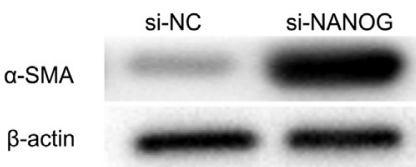

D

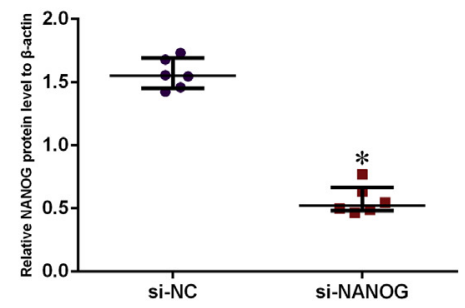

G

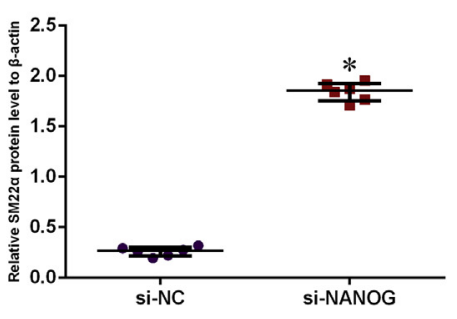

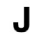

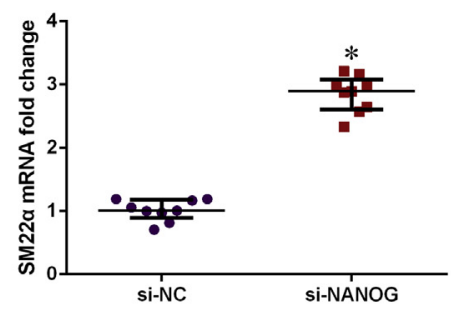

B

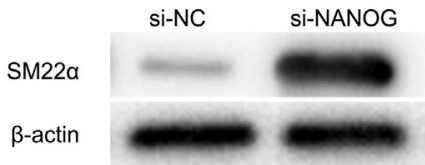

$\mathbf{E}$

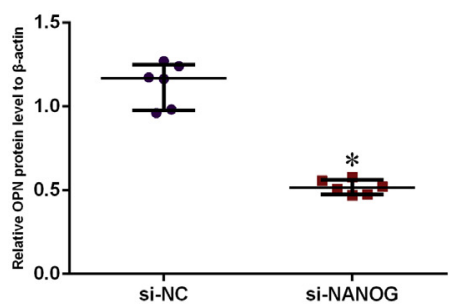

$\mathbf{H}$

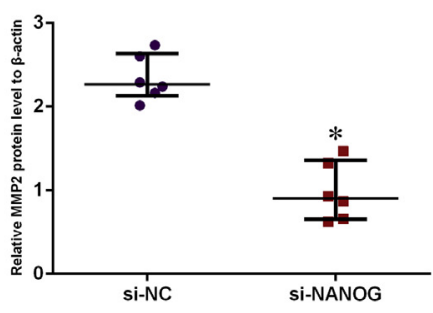

C
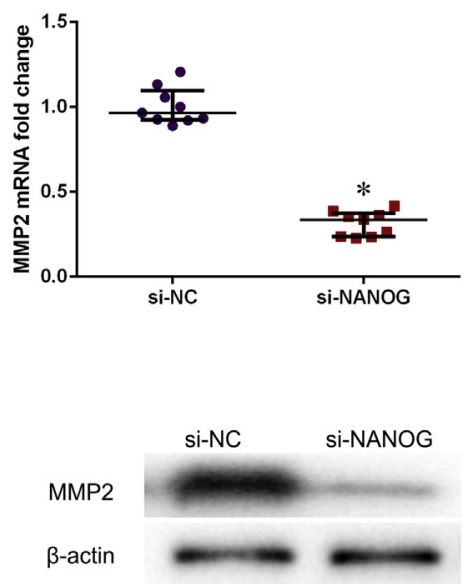

$\mathbf{F}$

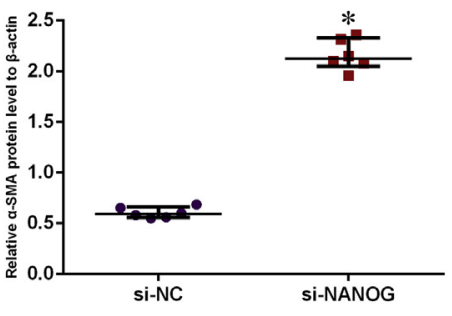

FIGURE E4. NANOG silence up-regulated VSMCs contractile markers and down-regulated VSMCs synthetic marker. A-C, VSMCs contractile markers (A) $\alpha$-SMA and B, SM22 $\alpha$ were increased whereas (C) VSMCs synthetic MMP2 was decreased in VSMCs transfected with si-NANOG on an mRNA level $(\mathrm{n}=9$ ). D-F, Protein level of VSMCs contractile markers $\alpha$-SMA (D and I) and SM $22 \alpha$ (E and J) were increased and the expression of VSMCs synthetic marker MMP2 ( $\mathrm{F}$ and $\mathrm{K}$ ) was decreased in si-NANOG VSMCs $(\mathrm{n}=6)$. $\mathrm{G}$ and H, Semiquantitative analysis for Western blot results in Figure 5 showed protein level of (G) NANOG and (H) OPN were decreased in si-NANOG VSMCs $(\mathrm{n}=6)$. $\beta$-actin was used as the internal reference in Western blot. VSMCs transfected with si-NC served as a control; error bars represent the median, $25 \%$ and $75 \%$ quartiles. Student $t$ test was used to compare differences between 2 groups. ${ }^{*} P<.05$ versus si-NC. $\alpha$-SMA, $\alpha$-smooth muscle actin; si-NC, scrambled negative control siRNA; NANOG, Nanog homeobox; si-NANOG, NANOG-specific small interfering RNA; SM22 $\alpha$, smooth muscle 22 $\alpha$; MMP2, matrix metalloproteinase; $O P N$, osteopontin. 

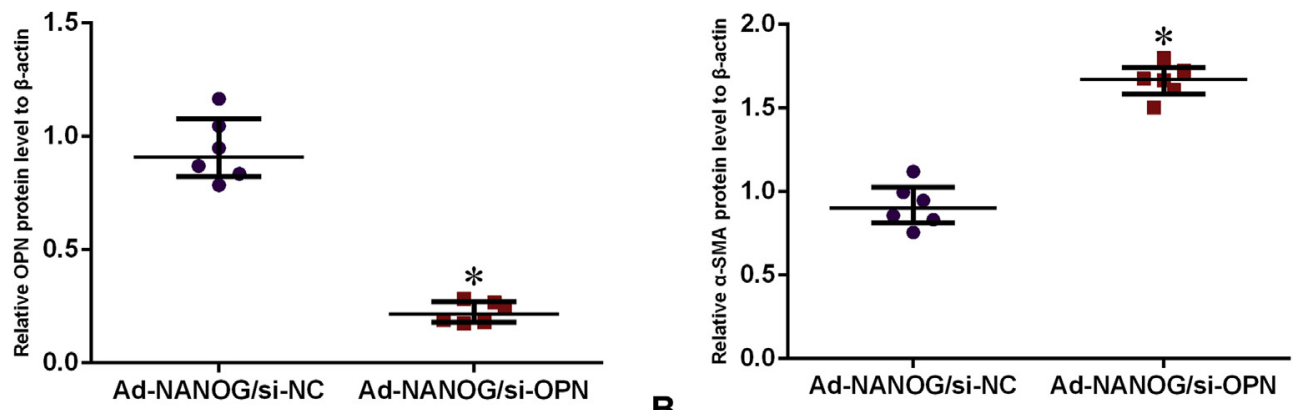

A

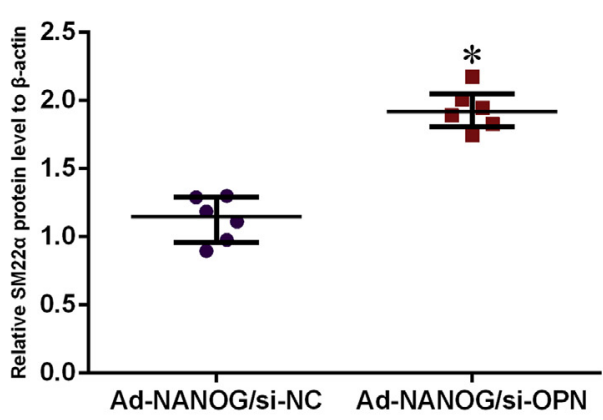

$\mathbf{B}$

C

Ad-NANOG/si-NC Ad-NANOG/si-OPN

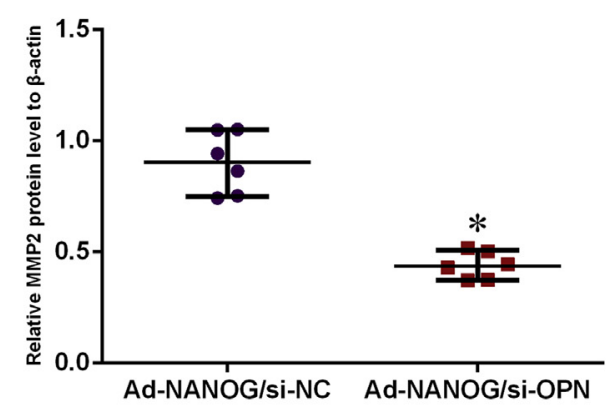

FIGURE E5. Semiquantitative analysis for Western blot results in Figure 6. Western blot semiquantitative results showed protein levels of (A) OPN and (D) MMP2 were decreased whereas (B) $\alpha$-SMA and (C) SM22 $\alpha$ were increased in VSMCs cotransfected with (Ad-NANOG/si-OPN) $(\mathrm{n}=6)$ compared with AdNANOG/scrambled negative control siRNA adenovirus Ad-NANOG/si-NC VSMCs $(n=6)$. Error bars represent the median, $25 \%$ and $75 \%$ quartiles. Student $t$ test was used to compare differences between 2 groups. $* P<.05$ versus Ad-NANOG/si-NC. OPN, Osteopontin; NANOG, Nanog homeobox; $A d-N A N O G / s i$-NC, NANOG overexpression adenovirus with scrambled negative control siRNA; Ad-NANOG/si-OPN, NANOG overexpression adenovirus with green fluorescent protein tag and OPN-specific small interfering RNA adenovirus; $\alpha$-SMA, $\alpha$-smooth muscle actin; $S M 22 \alpha$, smooth muscle $22 \alpha$; $M M P 2$, matrix metalloproteinase.

TABLE E1. Clinical details of patients in TAD and control group

\begin{tabular}{|c|c|c|c|}
\hline & Controls $(\mathbf{n}=10)$ & TAD $(\mathbf{n}=20)$ & $P$ value \\
\hline Age, y & $52.4 \pm 9.0$ & $54.6 \pm 9.8$ & .58 \\
\hline Sex, male & $7(70.0 \%)$ & $13(65.0 \%)$ & .78 \\
\hline BMI & $23.9 \pm 4.0$ & $25.7 \pm 3.6$ & .18 \\
\hline Hypertension & $7(70.0 \%)$ & $16(80.0 \%)$ & .54 \\
\hline LVEF, \% & $57.7 \pm 6.7$ & $59.5 \pm 6.2$ & .41 \\
\hline Diabetes mellitus & $1(10.0 \%)$ & $1(5.0 \%)$ & 1 \\
\hline MD of ascending aorta, $\mathrm{cm}$ & $2.44 \pm 0.66$ & $4.47 \pm 1.2$ & $<.001$ \\
\hline Smoke & $5(50.0 \%)$ & $5(25.0 \%)$ & .34 \\
\hline Renal dysfunction & 0 & 0 & \\
\hline Dyslipidemia & $2(20.0 \%)$ & $3(15.0 \%)$ & 1 \\
\hline Arteritis & 0 & 0 & \\
\hline Bicuspid aortic valve & 0 & 0 & \\
\hline Family history of aortic diseases & 0 & 0 & \\
\hline Aortic valve diseases & $10(100 \%)$ & $7(35.0 \%)$ & $<.001$ \\
\hline Calcific aortic valve disease & 3 & 0 & \\
\hline Rheumatic aortic valve disease & 6 & 0 & \\
\hline Aortic valve prolapse & 1 & 0 & \\
\hline Aortic valve rupture & 0 & 7 & \\
\hline Emergent operation & $0(0.0 \%)$ & $20(100.0 \%)$ & $<.001$ \\
\hline
\end{tabular}

Data presented as mean \pm standard deviation or $\mathrm{n}(\%) . P<.05$ was considered statistically significant. TAD, Thoracic aortic dissection; $B M I$, body mass index; $L V E F$, left ventricular ejection fraction; $M D$, maximum diameter. 